\title{
Abel ODEs: Equivalence and Integrable Classes
}

\author{
E.S. Cheb-Terrab ${ }^{a, b, c}$ and A.D. Roche ${ }^{d}$ \\ ${ }^{a}$ Department of Mathematics \\ University of British Columbia, Vancouver, Canada. \\ ${ }^{b}$ CECM, Department of Mathematics and Statistics \\ Simon Fraser University, Vancouver, Canada. \\ ${ }^{c}$ Department of Theoretical Physics \\ State University of Rio de Janeiro, Brazil. \\ ${ }^{d}$ Symbolic Computation Group \\ Computer Science Department, Faculty of Mathematics \\ University of Waterloo, Ontario, Canada.
}

Original version: 17 July 1999. Revised version: 5 January 2000

\begin{abstract}
A classification, according to invariant theory, of non-constant invariant Abel ODEs known as solvable and found in the literature is presented. A set of new integrable classes depending on one or no parameters, derived from the analysis of the works by Abel, Liouville and Appell [2, 3, 田, is also shown. Computer algebra routines were developed to solve ODEs members of these classes by solving their related equivalence problem. The resulting library permits a systematic solving of Abel type ODEs in the Maple symbolic computing environment.
\end{abstract}

\section{PROGRAM SUMMARY}

Title of the software package: Extension to the Maple ODEtools package

Catalogue number: (supplied by Elsevier)

Software obtainable from: CPC Program Library, Queen's University of Belfast, N. Ireland (see application form in this issue)

Licensing provisions: none

Operating systems under which the program has been tested: UNIX, Macintosh, Windows (95/98/NT).

Programming language used: Maple V Release 5

Memory required to execute with typical data: 16 Megabytes.

Keywords: Abel type first order ordinary differential equations (ODEs), equivalence problem, integrable cases, symbolic computation. 
Nature of mathematical problem

Analytical solving of Abel type first order ODEs having non-constant invariant.

Methods of solution

Solving the equivalence problem between a given ODE and representatives of a set of non-constant invariant Abel ODE classes for which solutions are available.

Restrictions concerning the complexity of the problem

The computational routines presented work when the input ODE belongs to one of the Abel classes considered in this work. This set of Abel classes can be extended, but there are classes - depending intrinsically on many parameters - for which the solution of the equivalence problem, as presented here, may lead to large and therefore untractable expressions. When the invariants of a given Abel ODE depend on analytic functions, the success of the routines depends on Maple's ability to normalize these invariants and recognize zeros (this is well implemented in Maple, but it may nevertheless not work as expected in some cases). Also, when the solution for the class parameter depends on other algebraic symbols entering the ODE being solved, the routines can determine this dependency only when it has rational form.

Typical running time

The methods being presented here have been implemented in the framework of the ODEtools Maple package. On the average, over Kamke's [1 first order Abel examples (see sec. 6), the ODE-solver of ODEtools is now spending $\approx 6$ sec. per ODE when successful, and $\approx 11$ sec. when unsuccessful. The timings in this paper were obtained using Maple R5 on a Pentium II 400 - 128 Mb. of RAM - running Windows98.

Unusual features of the program

These computational routines are able - in principle - to integrate the infinitely many members of all the nonconstant invariant Abel ODE classes considered in this work. Concretely, when a given Abel ODE belongs to one of these classes, the routines can determine this fact, by solving the related equivalence problem, and then use that information to return a closed form solution without requiring further participation from the user. The ODE families that are covered include, as particular cases, all the Abel solvable cases presented in Kamke's and Murphy's books, as well as the Abel ODEs member of other classes not previously presented in the literature to the best of our knowledge. After incorporating the new routines, the ODE solver of the ODEtools package succeeds in solving $97 \%$ of Kamke's first order examples. 


\section{LONG WRITE-UP}

\section{Introduction}

From some point of view, after Riccati type ODEs, the simplest first order ordinary differential equations (ODEs) are those having as right hand side (RHS) a third degree polynomial in the dependent variable, also called Abel type ODEs'

$$
y^{\prime}=f_{3} y^{3}+f_{2} y^{2}+f_{1} y+f_{0}
$$

where $y \equiv y(x)$, and $f_{0}, f_{1}, f_{2}$ and $f_{3}$ are analytic functions of $x$. As opposed to Riccati ODEs, for which integration strategies can be built around their equivalence to second order linear ODEs, Abel ODEs admit just a few available integration strategies, most of them based on the pioneering works by Abel, Liouville and Appell around 100 years ago [2, 3, 4]. In those works it was shown that Abel ODEs can be organized into equivalence classes. Two Abel ODEs are defined to be equivalent if one can be obtained from the other through the transformation

$$
\{x=F(t), \quad y(x)=P(t) u(t)+Q(t)\}
$$

where $t$ and $u(t)$ are respectively the new independent and dependent variables, and $F, P$ and $Q$ are arbitrary functions of $t$ satisfying $F^{\prime} \neq 0$ and $P \neq 0$.

Integration strategies were then discussed in [3, 4], around objects invariant under Eq.(2)] (herein called the invariants) which can be built with the coefficients $\left\{f_{3}, f_{2}, f_{1}, f_{0}\right\}$ and their derivatives. To each class there corresponds a different set of values of these invariants, and actually any one of them (we shall pick one and call it the invariant) is enough to characterize a class. A simple integrable case happens when the invariant is constant $t^{3}$; the solution to the ODE then follows straightforwardly in terms of quadratures, as explained in textbooks 迆, 司. On the contrary, when the invariant is not constant, just a few integrable cases are known and the formulation of solving strategies based on the equivalence between two such Abel ODEs, one of which is integrable, appears to be only partially explored in the literature, and not explored in general in computer algebra systems.

Having this in mind, this paper concerns Abel ODEs with non-constant invariant and presents:

1. A discussion and classification of the integrable Abel ODEs found both in Kamke's book and in the works from the late $19^{\text {th }}$ and early $20^{\text {th }}$ century by Abel, Appell, Liouville and other sources;

2. A set of new integrable Abel ODE classes - some depending on arbitrary parameters - derived from those aforementioned works;

3. An explicit method of verifying or denying the equivalence between two given Abel ODEs, one of which we know how to solve since it represents one of the above mentioned classes; and in the positive case, a way to determine the function parameters $F, P$ and $Q$ of the transformation Eq.(2) which maps one into the other;

\footnotetext{
${ }^{1}$ For convenience, in this work, by "Abel ODEs" we mean Abel ODEs of first kind, since Abel ODEs of second kind can always be transformed into first kind by a simple change of variables.

${ }^{2}$ The invariants change in form for $F(t) \neq t$, but keep their value. See Eq. (5).

${ }^{3}$ There exists one invariant such that if it is constant then the other invariants are as well.
} 
4. A computational scheme to resolve the equivalence problem in the case of parameterized classes, including the determination of the value for the class parameter when the equivalence exists;

5. A set of computer algebra (Maple) routines implementing the algorithms presented in items (3) and (4) above, to systematically solve - in principle - any Abel ODE belonging to one of the classes, parameterized or not, presented here and for which a closed form solution is known (items (1) and (2) above).

Item (11) is interesting since the Abel ODEs shown in textbooks in general, including Kamke's book, are displayed without further classification, and in fact many of them belong to the same class. This classification in terms of invariant theory is also necessary in a computational scheme for solving Abel ODEs as the one being presented, and we have not found it in other references. The integrable classes mentioned in (2) are new to the best of our knowledge, although directly or indirectly derived from previous works. The formulation of the equivalence problem mentioned in (3) is the one given by Liouville in [3], is systematic and does not involve solving any auxiliary differential equations . Concerning item (4), the idea can be viewed as a way of avoiding the untractable expressions which one would encounter when making direct use of Liouville's strategy with parameterized classes. The strategy presented is applicable when there exists a solution for some numerical values of the parameter, or when this parameter is a rational function of other symbols entering the input ODE. Regarding item (5), the implementation presented here is, as far as we know, unique in computer algebra systems in its ability to solve non-constant invariant, parameterized or not, Abel ODE classes.

The paper is organized as follows. In sec. 2, the basic definitions and the classic formulation of the equivalence problem in terms of invariants is reviewed and shown to apply straightforwardly to the case of a non-parameterized class. In sec. [3 it is shown how these ideas can be complemented by taking advantage of computers to tackle the equivalence problem in the case of a parameterized class. Section 4 presents a classification of the integrable classes we have found in the literature with some additional comments as to their derivation. In sec. 5 new integrable Abel classes are presented. In sec. 6, a test-suite for the routines presented is discussed and statistics are shown describing the performance with this test-suite as well as with Kamke's first order examples. Finally, the conclusions contain some general remarks about this work and its possible extensions.

Additionally, we present in the Appendix a table listing the distinct Abel ODE classes that we have found, representative ODEs from each class, and their respective solutions.

\section{Classical Theory for Abel ODEs}

In general, Abel type ODEs can be studied using two related concepts: invariants and ODE equivalence classes. We define two Abel ODEs to be equivalentP if one can be obtained from the other using a transformation of the form Eq.(2). The equivalence class containing a given ODE is then the set of all the ODEs equivalent to the given one. We note that although the infinitely many members of a class can be mapped into each other by using Eq.(2), there are also infinitely many disjoint Abel classes (Eq.(2) is not sufficient to map any Abel ODE into a given one).

To each class one can associate an infinite sequence of absolute invariants [3, 过. To see this, consider two Abel ODEs, the first Eq.(1), the second obtained from Eq.(1) through the transformation

\footnotetext{
${ }^{4}$ An approach somewhat similar to this one by Liouville is discussed in $[6]$.

${ }^{5}$ For a more formal definition of class see 7
} 
Eq.(2)

$$
u^{\prime}=\tilde{f}_{3} u^{3}+\tilde{f}_{2} u^{2}+\tilde{f}_{1} u+\tilde{f}_{0}
$$

where the coefficients $\left\{\tilde{f}_{0}, \tilde{f}_{1}, \tilde{f}_{2}, \tilde{f}_{3}\right\}$, are related to the those of Eq.(1) by

$$
\begin{aligned}
& \tilde{f}_{0}=\frac{F^{\prime}\left(f_{0}(F)+f_{1}(F) Q+f_{2}(F) Q^{2}+f_{3}(F) Q^{3}\right)-Q^{\prime}}{P} \\
& \tilde{f}_{1}=\frac{P^{\prime}}{P}-F^{\prime}\left(f_{1}(F)+2 f_{2}(F) Q+3 f_{3}(F) Q^{2}\right) \\
& \tilde{f}_{2}=P F^{\prime}\left(f_{2}(F)+3 f_{3}(F) Q\right) \\
& \tilde{f}_{3}=P^{2} F^{\prime} f_{3}(F)
\end{aligned}
$$

Following [4], we call an absolute invariant of Eq.(11) a function $I(f, x)$ of the coefficients $\left\{f_{0}, f_{1}, f_{2}, f_{3}\right\}$ and their derivatives with respect to $x$ such that, for all $\{F, P, Q\}$ in Eq.(2),

$$
\left.I(\tilde{f}, t)\right|_{\tilde{f}=\tilde{f}(f, t)}=\left.I(f, x)\right|_{x=F(t)}
$$

where $\tilde{f}=\tilde{f}(f, t)$ represents the coefficients $\left\{\tilde{f}_{0}, \tilde{f}_{1}, \tilde{f}_{2}, \tilde{f}_{3}\right\}$ and their derivatives with respect to $t$, expressed using Eq.(4).

Similarly, we call a relative invariant a function, say $s$, of the coefficients of Eq.(11) and their derivatives such that when changing variables using Eq.(2), the resulting expression is equal to the original one up to a factor, say $\varphi_{s}$, dependent uniquely on the functions $F, P$, and $Q$ in Eq.(2) and independent of the coefficients themselves [7]:

$$
\left.s(\tilde{f})\right|_{\tilde{f}=\tilde{f}(f, t)}=\left.\varphi_{s}(F, P, Q) s(f)\right|_{x=F(t)}
$$

Liouville showed that in the case of Abel equations there is a relative invariant of weight 3

$$
s_{3} \equiv f_{0} f_{3}^{2}+\frac{1}{3}\left(\frac{2 f_{2}^{3}}{9}-f_{1} f_{2} f_{3}+f_{3} f_{2}^{\prime}-f_{2} f_{3}^{\prime}\right)
$$

which can be used to recursively generate an infinite sequence of relative invariants $s_{2 m+1}$ of weights $2 m+1$ respectively[ through the formula

$$
s_{2 m+1} \equiv f_{3} s_{2 m-1}^{\prime}-(2 m-1) s_{2 m-1}\left(f_{3}^{\prime}+f_{1} f_{3}-\frac{f_{2}^{2}}{3}\right)
$$

As is clear from this definition, the product of two relative invariants respectively of weights $n$ and $m$ is a relative invariant of weight $n+m$, and by dividing any two relative invariants of equal weight one can generate an infinite sequence of absolute invariants

$$
I_{1}=\frac{s_{5}^{3}}{s_{3}^{5}}, I_{2}=\frac{s_{7} s_{3}}{s_{5}^{2}}, I_{3}=\frac{s_{9}}{s_{3}^{3}}, \text { etc } \ldots
$$

\footnotetext{
${ }^{6}$ In the case of $s_{3}, \varphi_{s_{3}}=\left(F^{\prime} P\right)^{3}$; the weight $n$ refers to the degree of $\varphi_{s_{n}}$ with respect to $\left(F^{\prime} P\right)$.
} 
In [4], Appell showed that this sequence can also be obtained from two basic absolute invariants - say $J_{1}, J_{0}$, by expressing $J_{1}$ as a function of $J_{0}$ and then differentiating the result with respect to $J_{0}$. As a consequence, if $I_{1}$ is constant then all the other ones will be too. This fact allows one to identify the constant character of the invariants in Eq.(9) by looking at just the first one. We note also that there are infinitely many different classes having $I_{1}$ constant, related to the infinitely many possible constant values $I_{1}$ can have.

\subsection{Integration strategy}

A description of a method of integration when the invariants are constant 7 is found in the works by Abel [2], Liouville [3] and Appell [4]. In such a case, all members of the class can be systematically mapped into a separable first order ODE (representative of the class), by appropriately choosing $F, P$ and $Q$ in Eq.(2); see for instance [1] and [5].

A quite different situation happens when $I_{1}$ is not constant. In such a case, relatively few integrable Abel ODEs are known, and the integration methods used to solve each of them depend in an essential way on non-invariant properties of the coefficients $f$. Those methods are then useless for solving the other infinitely many members of the same classes, unless one can solve the related equivalence problems; i.e., determining - when they exist -the values of $F, P$ and $Q$ in Eq.(2) linking two Abel ODE which belong to the same class.

\subsection{Identifying an ODE as member of a given Abel ODE class}

Consider two Abel ODEs; the first one given by Eq.(11), and a second one being of the same form, but with coefficients $\tilde{f}_{0}, \tilde{f}_{1}, \tilde{f}_{2}$ and $\tilde{f}_{3}$. The problem now is to determine whether the second Abel ODE can be obtained from Eq.(1) by changing variables using Eq.(2). This problem can be formulated by equating the coefficients between the transformed equation, obtained by applying the transformation Eq.(2) to Eq.(11), and the second Abel ODE, resulting in Eq.(14), which can be seen as an ODE system for $\{F, P, Q\}$. To solve this system, following Liouville [3], we first note that the absolute invariants corresponding to the two Abel ODEs don't depend on $P$ or $Q$ (see previous section). Hence the function $F$ entering Eq.(2) can be obtained by just running an elimination process using two of these absolute invariants, for instance $I_{1}=s_{5}^{3} / s_{3}^{5}$ and $I_{2}=s_{5} s_{7} / s_{3}^{4}$ :

$$
0=\frac{\tilde{s}^{3}}{{\tilde{s_{3}}}^{5}}-\left.\frac{s_{5}^{3}}{s_{3}^{5}}\right|_{x=F(t)} \quad 0=\frac{\tilde{s_{5}} \tilde{s_{7}}}{{\tilde{s_{3}}}^{4}}-\left.\frac{s_{5} s_{7}}{s_{3}^{4}}\right|_{x=F(t)}
$$

As discussed in [3, 4], the existence of a common solution $F(t)$ to both equations above (such that $F^{\prime} \neq 0$ ) is the necessary and sufficient condition for the existence of a transformation Eq.(2) relating the two Abel ODEs. Once $F$ is known, the system Eq.(4) becomes trivial in that $P$ and $Q$ can be re-expressed in terms of $F$ by performing fairly simple calculations. In the case of interest of this work - non-constant invariant

$$
P(t)=\left.\frac{F^{\prime}{\tilde{f_{3}}}^{2} s_{3}}{f_{3}^{2} \tilde{s_{3}}}\right|_{x=F(t)} \quad Q(t)=\left.\frac{F^{\prime} \tilde{f}_{2} \tilde{f}_{3} s_{3}-f_{2} f_{3} \tilde{s_{3}}}{3 f_{3}^{2} \tilde{s}_{3}}\right|_{x=F(t)}
$$

where $\left\{f_{i}, \tilde{f}_{i}\right\}$ with $i: 0 \rightarrow 3$ are the coefficients of the two Abel equations, $s_{3}$ is the relative invariant Eq.(7) expressed in terms of $f_{i}$ and $\tilde{s_{3}}=\left.s_{3}\right|_{f_{i}=\tilde{f}_{i}}$.

\footnotetext{
${ }^{7}$ In [6] it is also shown that in the constant invariant case the problem can also be formulated in terms of the symmetries of these ODEs.

${ }^{8}$ When the invariant is non-constant, $s_{3} \neq 0$.
} 
Concerning the explicit solution $F(t)$ for Eq.(10), we note that our interest in solving the equivalence problem is in that it leads directly to the solution of other members of an Abel class, when the solution to a representative of the class is known. In turn, all the solvable classes we are aware of have a representative with rational coefficients (see the Appendix), and hence also rational invariants 5 . Hence, assuming that one of the two Abel ODEs has rational coefficients and that Eq.(10) was obtained by applying Eq.(2) to it, the system Eq.(10) will always be polynomial in $F(t)$. In such a case, when a common solution $F(t)$ to both equations exists, the resultant between these polynomials will be zero [3]; i.e.: there will be a common factor, depending on $F$ and $t$ and representing the common solution, which can be obtained by calculating the greatest common divisor (GCD) between the two equations in Eq.(19). Conversely, if that GCD does not depend on $F$, a transformation Eq.(2) linking the input equation to Eq.(11) does not exist. That the dependence on $F$ of this GCD is a necessary condition for the existence of the desired transformation Eq.(2) is a consequence of the validity of Eq.(5) and hence the system (10). A proof of its sufficiency was given by Appell in 《4].

The whole process just described to determine the equivalence between two given Abel ODEs, one of which is rational in $x$, can be summarized as follows:

1. Calculate two absolute invariants, set up the system Eq.(10), and calculate the GCD between the two equations;

2. When this GCD does not depend on $F$, the ODEs don't belong to the same class; otherwise determine an explicit expression for $F(t)$ from the result of the GCD calculation;

3. Plug this value for $F$ into the formulas Eq.(11) to determine the values of $P(t)$ and $Q(t)$, arriving in this way at the transformation Eq.(2) mapping one Abel ODE into the other.

Example:

Consider the two non-constant invariant Abel ODEs

$$
\begin{gathered}
y^{\prime}=-\frac{1}{2(x+4)}\left(x y^{3}+y^{2}\right) \\
y^{\prime}=\frac{\left(f^{\prime} x-f\right)}{2(f+3 x)}\left((x-f) y^{3}+y^{2}\right)-\frac{y}{x}
\end{gathered}
$$

where in the above $f \equiv f(x)$ is an analytic (arbitrary) function. As in the typical situation one of these ODEs has invariants rational in $x$ and we know its solution; i.e. for Eq.(12) we have

$$
C_{1}+\frac{\sqrt{y^{2} x-4 y-1}}{y}+2 \arctan \left(\frac{1+2 y}{\sqrt{y^{2} x-4 y-1}}\right)=0
$$

where $C_{1}$ is an arbitrary constant. We would then like to determine whether there are functions $\{F, P, Q\}$ so that Eq.(12) transforms under Eq.(2) into Eq.(13), and if so, determine $\{F, P, Q\}$ and use them together with Eq.(14) to build the answer to Eq.(13). For this purpose, we start (step (11)) by computing the relative invariants $s_{3}, s_{5}, s_{7}$, leading to Eq.(10)

\footnotetext{
${ }^{9}$ On the other hand, there is no reason to expect that the second of the two Abel ODEs being tested for equivalence also has rational invariants. If however the invariants of both Abel ODEs are rational in $t$ and the coefficients are numbers, then it is also possible to determine $F(t)$ by performing a rational function decomposition as mentioned in 6.
} 


$$
\begin{aligned}
& 0=\frac{\left(87 f t+9 f^{2}+184 t^{2}\right)^{3}}{t(9 f+31 t)^{5}}-\frac{\left(280+105 F+9 F^{2}\right)^{3}}{(40+9 F)^{5}} \\
& 0=\frac{\left(81 f^{3}+1431 f^{2} t+7185 f t^{2}+10903 t^{3}\right)(9 f+31 t)}{\left(87 f t+9 f^{2}+184 t^{2}\right)^{2}}-\frac{\left(1674 F^{2}+10290 F+81 F^{3}+19600\right)(40+9 F)}{\left(280+105 F+9 F^{2}\right)^{2}}
\end{aligned}
$$

where in the above $F \equiv F(t)$ is the function we are looking for and $f$ is taken at $x=t$. We recall that when Eq.(13) has non-constant invariant the denominators in above are not zero since $s_{3} \neq 0$. Calculating the GCD between the numerators of the expressions above (step (2)) and equating this GCD to zero, we obtain

$$
27(t+t F-f)=0
$$

from where the common solution $F(t)$ to both equations is given by

$$
F(t)=\frac{f(t)}{t}-1
$$

Substituting this value of $F$ into Eq.(11), a transformation of the form Eq.(2) mapping Eq.(12) into Eq.(13) is finally given by

$$
\left\{x=\frac{f(t)}{t}-1, \quad y(x)=t u(t)\right\}
$$

from where by changing variables in Eq.(14) using the transformation above and renaming the variables $(t \rightarrow x, u \rightarrow y)$, the solution to Eq.(13) is obtained

$$
C_{1}+\frac{\sqrt{\left(\frac{f}{x}-1\right) x^{2} y^{2}-4 x y-1}}{x y}+2 \arctan \left(\frac{(1+2 x y)}{\sqrt{\left(\frac{f}{x}-1\right) x^{2} y^{2}-4 x y-1}}\right)=0
$$

\section{Parameterized Abel ODE classes}

We formulate here the equivalence problem in the case of parameterized classes. By "parameterized class" we mean an (Abel) ODE class depending on symbolic parameters which cannot be removed by changing variables using Eq.(2). The interest in parameterized solvable classes is clear: to each set of values of the parameters corresponds - roughly speaking - a different Abel class $\boxplus$. Hence, a formulation of the equivalence problem for parameterized classes enables one to solve all the members of infinitely many classes at once.

In order to simplify the discussion, we consider the problem of an Abel ODE class depending on just one parameter $[\mathbb{Z}$, say $\mathcal{C}$. Also, we distinguish between two different types of problems: one is when the equivalence problem has a solution for a specific numerical value of $\mathcal{C}$; the other happens when to have a solution it is required that $\mathcal{C}$ assumes symbolic values, for instance in terms of other symbols entering the input ODE. We discuss first the numerical case, and in the next subsection we show how the symbolic case can be mapped into many numerical problems - when the parameter depends on other symbols in a rational manner - by using rational interpolation methods.

\footnotetext{
${ }^{10}$ We note that when $f(t)$ is an algebraic mapping involving varied analytic functions, Maple's procedures to simplify and put them in normal form may fail and consequently the GCD computation may not be successful.

${ }^{11}$ There may be particular different sets of values for which the resulting ODEs will nevertheless belong to the same class.

${ }^{12}$ The integrable classes presented in the literature depend at most on one parameter (see sec. 4).
} 


\subsection{Solution for some numerical value of $\mathcal{C}$}

To facilitate the exposition we present the discussion around a concrete example. Consider the equivalence problem between a given Abel ODE, for instance,

$$
y^{\prime}=8 \frac{\left(1-x^{4}-x^{8}\right) y^{3}}{x^{7}}+4 \frac{y^{2}}{x^{4}}+\frac{y}{x}
$$

and the one presented in Abel's memoires [2]

$$
y^{\prime}=\frac{\left(\mathcal{C} x^{4}+x^{2}+1\right) y^{3}}{x^{3}}+y^{2}
$$

If this equivalence exists, then it exists for a specific value of the parameter $\mathcal{C}$ since there is no solution for arbitrary $\mathcal{C}$ (the existence of such a solution would mean the class does not really depend on any parameter). Hence, the common solution $F(t)$ to the system Eq.(10) will not show up until the correct value of $\mathcal{C}$ is determined $[3$, invalidating the itemized algorithm of the previous section.

A natural alternative to this problem would be to take one more absolute invariant, for instance, $s_{3} s_{7} / s_{5}^{2}$, so that our system Eq.(10) becomes

$$
0=\frac{\tilde{s 5}^{3}}{\tilde{s_{3}}}-\left.\frac{s_{5}^{3}}{s_{3}^{5}}\right|_{x=F(t)} \quad 0=\frac{\tilde{s_{3}} \tilde{s_{7}}}{\tilde{s_{5}}}-\left.\frac{s_{3} s_{7}}{s_{5}^{2}}\right|_{x=F(t)} \quad 0=\frac{\tilde{s_{5}} \tilde{s_{7}}}{\tilde{s_{3}}{ }^{4}}-\left.\frac{s_{5} s_{7}}{s_{3}^{4}}\right|_{x=F(t)}
$$

and search for a solution to this problem such that $F^{\prime} \neq 0, \mathcal{C}^{\prime}=0$. For that purpose, eliminate $\mathcal{C}$ from the first and second expressions above by taking the resultant with respect to $\mathcal{C}$, obtaining - say - $R_{1}$. In the same way, eliminate $\mathcal{C}$ from the first and the third expressions of Eq.(22) obtaining $R_{2}$. Hence, when a solution exists, the resultant between $R_{1}$ and $R_{2}$ with respect to $F$ will vanish. In other words, the algorithm of the previous section will work if instead of performing the calculations over the expressions Eq.(10) we perform them over $R_{1}$ and $R_{2}$. The GCD between $R_{1}$ and $R_{2}$ will then return the factor depending on both $F$ and $t$, whose solution is the function $F(t)$ we are interested in.

This method, simple and correct in theory, unfortunately does not work in practice because the expressions tend to grow in size so much that the computation of the first of these three resultants may not be possible, even with a simple example such as the one shown above. The problem resides in the fact that multivariate GCDs and resultants are quite expensive operations for the current symbolic computation environments.

An alternative to this problem consists of reducing it to a sequence of bivariate GCD and resultant calculations, for which the available algorithms are relatively fast. The idea can be summarized as follows.

1. From the previous considerations, when a solution to the equivalence problem exists, the resultant between any two of the expressions in Eq.(22) will not vanish for any value of $t$, since we haven't introduced the correct (unknown at this point) value of $\mathcal{C}$. Hence, if we insert in Eq.(22) a numerical value ${ }^{\text {T4 }}$ for $t$ and calculate the GCD between any two of the resulting expressions, this GCD cannot contain any factor depending on $F$. This gives us a first "existence condition" test for the solution before proceeding further;

\footnotetext{
${ }^{13}$ There may be more than one solution $\mathcal{C}$.

${ }^{14}$ We note there may exist "invalid evaluation points"; roughly speaking to avoid this problem this evaluation point must not cancel any of the coefficients of the variables remaining in the system - see [8].
} 
2. When Eq.(22) evaluated at $t=$ number passed the test of the previous step, take two of the resulting three expressions and calculate their resultant with respect to $F$, obtaining, say, $\tilde{R}_{1}$. Then take a different pair and calculate their resultant with respect to $F$ again, obtaining, say, $\tilde{R}_{2}$. Neither of these resultants will vanish since the GCD calculations of the previous step showed no factor depending on $F$. Also, the calculation of $\tilde{R}_{1}$ and $\tilde{R}_{2}$ is now quite simpler since the expressions do not involve $t$;

3. Then if a solution to the problem exists, the GCD between $\tilde{R}_{1}$ and $\tilde{R}_{2}$ will yield a factor depending on $\mathcal{C}$; equating it to zero and solving it for $\mathcal{C}$ will give the common solution $\mathcal{C}$ for $\tilde{R}_{1}$ and $\tilde{R}_{2}$. More precisely, what we will get in this way is a set of candidates (including among them the correct value) for $\mathcal{C}$; not all of them will necessarily lead to a solution $F(t)$ to the original problem;

4. We now plug these candidates for $\mathcal{C}$ into Eq.(22), one at a time, receiving a system of three expressions involving again only two unknowns, now $F$ and $t$. If there is a common solution $F(t)$ to these expressions, the resultant with respect to $\mathrm{F}$ between any two of them will vanish. Hence, the GCD between those two expressions will contain a factor depending both on $F$ and $t$; equating this factor to zero and solving for $F$ leads to the solution $F(t)$.

Returning to our example of determining the equivalence between Eq.(20) and Eq.(21), the itemized procedure just outlined runs as follows.

According to step (11), $t=0$ is tried first, but it is found to be an invalid evaluation point. The next value of $t$ to try, $t=1$ turns out to be valid, so Eq.(22) was evaluated at $t=1$; the GCDs between any two of the three resulting expressions do not depend on $F$, so this first test for the "existence" of a solution passed.

Continuing with step (2), the calculation of $\tilde{R}_{1}$ and $\tilde{R}_{2}$ is performed without problems concerning the size of the expressions.

The GCD of step (3) results in the three factors: $36 \mathcal{C}-5, \mathcal{C}+1$ and $9 \mathcal{C}-2$; equating them to zero and solving them for $\mathcal{C}$ we arrive at three candidates for $\mathcal{C}$.

In step (四), plugging each of these candidates one at a time into Eq.(22) and taking the GCD between two of the three resulting expressions we note that $\mathcal{C}=5 / 36$ does not lead to any factor depending on both $F$ and $t$, but $\mathcal{C}=-1$ leads to such factor: $F^{2} t^{4}-1$. So that for $\mathcal{C}=-1$ the problem admits two solutions: $F= \pm 1 / t^{2}$.

Finally, by introducing $F=1 / t^{2}$ into the formulas for $P$ and $Q$ Eq.(11) we arrive at the transformation of the form Eq.(2) mapping Eq.(21) into Eq.(20)

$$
\left\{x=\frac{1}{t^{2}}, y=-2 \frac{u(t)}{t}\right\}
$$

and hence by applying the same change of variables to the answer of Eq.(21) and substituting $C=-1$ we obtain the answer to Eq.(20).

A remark however is in order: if, in step (3) of the algorithm just described, the numeric candidates for $\mathcal{C}$ involve fractional powers of rational numbers, the Maple system may then enter not efficient expensive computations, exhausting the system resources before determining the expression $F(t)$ solving the problem in step (四). The root of this limitation seems to be in the absence in Maple of built-in normalization for such "numeric radicals"

\footnotetext{
${ }^{15} \mathrm{~A}$ built-in normalization of radicals is implemented in the computer algebra system "Mathematica". For typical problems we tried where Maple exhausted the system resources trying to determine the solution $F(t)$, we exported
} 


\subsection{Solution when the parameter $\mathcal{C}$ is some rational function of other symbols}

When $\mathcal{C}$ assumes symbolic values, for instance it depends on other symbols - say $\{\alpha\}$ - entering the input ODE, if this dependency is rational it is possible to map the determination of $\mathcal{C}(\alpha)$ into a sequence of problems having for solution a numerical value of $\mathcal{C}$. In turn each of these numerical problems can be tackled using the algorithm of the previous subsection. The idea consists of attributing numerical values to the symbols $\{\alpha\}$ entering the invariants in order to determine $\mathcal{C}(\alpha)$ by means of a rational interpolation. To simplify the presentation we first discuss the case when $\{\alpha\}$ consists of a single parameter, and then show how to extend the algorithm to the case in which $\{\alpha\}$ consists of many parameters by commenting on a concrete example. So when $\alpha$ consists of just one parameter this rational interpolation scheme is summarized as follows:

1. Take the system Eq.(22) and attribute a numerical value to $\alpha$ (check for possible wrong evaluation points), so the resulting system depends on just $x, \mathcal{C}$ and $F$;

2. Enter step (11) in the algorithm of the previous subsection and run all the steps:

(a) If there is no solution for $\mathcal{C}$ and $F$ such that $\mathcal{C}^{\prime}=0$ and $F^{\prime} \neq 0$ then quit the process - the input ODE does not belong to this class, or the solution involves a non rational dependency of $\mathcal{C}$ on $\{\alpha\}$.

(b) If however a solution for $\mathcal{C}$ and $F$ was found, record the values for $\mathcal{C}$ and $\alpha$ - they represent a point of a curve $\mathcal{C}(\alpha)$

3. Using the points recorded so far, interpolate $\mathcal{C}$ as a function of $\alpha$ and test if this interpolated value already solves the problem for an arbitrary a 1 :

(a) If so, the problem has been solved;

(b) Otherwise change the evaluation point of $\alpha$ and re-enter step (2) of this enumeration;

We note that the rational interpolation of $\mathcal{C}(\alpha)$ requires the knowledge a priori of the polynomial degrees in $\alpha$ of both numerator and denominator. That information is not available in advance, but we know that these two degrees sum to $n-1$, where $n$ is the number of points being interpolated. So, when performing the test in step (3) and before going to step (3b) we actually test all possible different interpolations, starting with the maximum possible degree for the numerator and finishing with the maximum possible degree for the denominator.

Concerning the extension of this algorithm for the case when $\{\alpha\}$ involves more than one parameter, for instance $\{a, b\}$, this extension is easy and better illustrated with an example. Consider the equivalence problem between the Abel ODEs

$$
y^{\prime}=\frac{C\left(2 x^{2} C-2\right) y^{3}-3 C y^{2}+C x y}{1-x^{2} C} \text { and } y^{\prime}=\frac{a\left(2 x^{2} a-2 b\right) y^{3}-3 a y^{2} b+a b x y}{b\left(b-x^{2} a\right)}
$$

which solution is just the identity $x \rightarrow x, y \rightarrow y$, but only exists when $\mathcal{C}=\frac{a}{b}$ (we choose the identity without loss of generality and so that the solving process is easy to follow). We want to determine $\mathcal{C}=\frac{a}{b}$ (rational function of two parameters) using the algorithm just described.

the mathematical expressions involving radicals to Mathematica and noticed that $F(t)$ was determined in this other computer algebra system in reasonable time.

${ }^{16}$ This is done by restoring the symbol " $\alpha$ " in the system obtained in step (1) and checking if the system is satisfied. 
We start with step (1), take the system Eq.(22) corresponding to Eq.(24) and attribute numerical values for the first parameter, $a$, checking for possible wrong evaluation points - we end up evaluating this system at $a=1$. However, the system still depends on the second parameter, $b$, so we attribute numerical values to $b$ too - check for possible wrong evaluation points - and hence end up evaluating the system altogether at $a=1, b=2$. The resulting system now only depends on $x, F$ and $\mathcal{C}$.

So we enter step (2) (actually the whole algorithm of sec. 3.1) with this evaluated system of the previous step and detect that $\mathcal{C}=1 / 2$ leads to the solution $F=x$; so a solution $\mathcal{C}^{\prime}=0$ and $F^{\prime} \neq 0$ exists.

Hence, in step (3) we interpolate $\mathcal{C}(b)$ (so far we have just one point, so $\mathcal{C}(b)=1 / 2$ ) and test this value for arbitrary $b$, verifying that the interpolation is still incomplete: the system Eq.(22) with $a=1$ and arbitrary $b$ is not satisfied by $\mathcal{C}=1 / 2, F=x$.

We are then in step (3.3b); so attribute a new numerical value to $b$, hence evaluate the system Eq.(22) at $a=1, b=3$ and re-enter step (2) finding that $\mathcal{C}=1 / 3$ leads to $F=x$.

We record this new point (step (2.2b) ) and interpolate - now using the two points obtained so far - our first interpolation is of degree 1 in $b: \mathcal{C}(b)=(5-b) / 6$. This interpolation however does not solve the problem for arbitrary $b$. So we increase by one the degree in $b$ of the denominator in the interpolation, resulting in $\mathcal{C}(b)=1 / b$, and verify that this second interpolation indeed solves the system for arbitrary $b$ - so the interpolation for $b$ at $a=1$ is complete.

We return then to the interpolation of $\mathcal{C}(a, b)$ with respect to $a$, record that for $a=1$ there is a solution $\mathcal{C}(b)=1 / b$, and test this solution for arbitrary a (step (3) with respect to $a$ ), verifying that the interpolation for $a$ is still incomplete.

Hence we re-enter step (四) with new evaluation points $a=2, b=4$, and re-start the process of determining $\mathcal{C}(b)$.

Following the same steps just described, for $a=2$ we find that $\mathcal{C}(b)=2 / b$ solves the problem for arbitrary $b$, leading to the second point in the interpolation of $\mathcal{C}(a, b)$ with respect to $a$.

So we are now in step (3) again, and interpolating $\mathcal{C}$ using the two points obtained so far we find $\mathcal{C}(a, b)=a / b$ - this value of $\mathcal{C}(a, b)$ is verified to satisfy the system Eq.(22) for arbitrary $a$, and thus the problem has been solved.

The algorithm just described, though expensive in computations, is successful in solving the equivalence problem in reasonable time for typical situations (see sec. 6). In this example, for instance, it took 12 seconds to: setup the invariants and the system Eq.(22), simplify this system to a normal form, run all the items of the algorithm just described to determine $\mathcal{C}(a, b)$ and $F(t)$, then determine $P(t)$ and $Q(t)$ according to Eq.(11), and finally return a solution to the second of the ODEs in Eq.(24).

\subsubsection{Remark on the existence of multiple solutions for the class parameter $\mathcal{C}(\alpha)$}

The interpolation algorithm just described is valid provided there is only one solution curve $\mathcal{C}(\alpha)$; otherwise we may end up trying to interpolate $\mathcal{C}(\alpha)$ using points which belong to different solution curves, leading nowhere. In turn, the existence of many curves $\mathcal{C}(\alpha)$ solving a given problem is related to the existence of symmetries in the invariants (of the ODE representative of the class we want to match) entering Eq.(22). Concretely, if the mapping $\{\mathcal{C} \rightarrow \kappa(\mathcal{C}), x \rightarrow \phi(x, \mathcal{C})\}$ is a symmetry of these invariants, then if $\{\mathcal{C}, F(x)\}$ leads to a solution for the equivalence problem, consequently $\{\kappa(\mathcal{C}), \phi(F(x), \mathcal{C})\}$ will also lead to a (different) solution. A concrete example of this situation is discussed in sec. 6.1.

Concerning detecting this situation, we note that if the mapping $\{\mathcal{C} \rightarrow \kappa(\mathcal{C}), x \rightarrow \phi(x, \mathcal{C})\}$ is a symmetry of the invariants, then the inverse mapping is also a symmetry, and since these invariants 
are rational in both $x$ and $\mathcal{C}$, the form of such a symmetry mapping is

$$
\kappa(\mathcal{C})=\frac{a \mathcal{C}+b}{c \mathcal{C}+d}, \quad \phi(x, \mathcal{C})=\frac{f(\mathcal{C}) x+g(\mathcal{C})}{h(\mathcal{C}) x+j(\mathcal{C})}
$$

that is, a fractional linear mapping, where $\{a, b, c, d\}$ and $\{f(\mathcal{C}), g(\mathcal{C}), h(\mathcal{C}), j(\mathcal{C})\}$ are in principle constants and functions to be determined. Regarding the use of interpolation methods, the problem happens when $\kappa(\mathcal{C}) \neq \mathcal{C}$. A first manner of detecting this problem then consists of setting up the system of equations and inequations

$$
I(x, \mathcal{C})=I(x, \mathcal{C})_{\substack{x=\phi(x, \mathcal{C}) \\ \mathcal{C}=\kappa(\mathcal{C})}}, \quad \phi=\frac{F}{G}, \quad F_{x x}=0, \quad G_{x x}=0, \quad \kappa(\mathcal{C}) \neq \mathcal{C}
$$

where $I(x, \mathcal{C})=s_{5}^{3} / s_{3}^{5}$ is the first invariant and $F, G$ and $\kappa$ are the unknowns to be determined, and seeing if this system is consistent. This check for consistency can be performed by simplifying this system with respect to its integrability conditions - for this purpose we used the diffalg [13] and RIF [14] Maple packages. When the system has no solution, this fact is detected by these packages; otherwise the related symmetry of the invariants is obtained from the output of these packages directly.

\section{Integrable Abel ODE classes found in the literature}

This section is devoted to a compilation of integrable Abel ODE classes found in the literature. The compilation is not intended to be complete, but it nevertheless covers various of the usual references; mainly Kamke's and Murphy's books [1, 5], and the original works by Abel, Liouville and others on these subjects [2, 3, 4, 10].

One of the noticeable things in these references is that the presentation of integrable cases lacks a classification in terms of their invariants. Consequently, many of these ODEs can actually be obtained from one another by means of Eq.(2), that is, they belong to the same class. Since part of this work consisted in writing computer routines addressing the equivalence problem, we performed this classification, and therefore present here a more compact collection of integrable Abel ODE classes, as opposed to just integrable ODEs. Classes not depending on parameters are labelled by numbers (e.g., Class 1), while those depending on parameters are labelled with letters (e.g., Class A). The solutions to the representatives of these classes are presented altogether in a table in the Appendix.

While revising the related literature we also noticed that various of the cases presented in books or papers are in fact particular cases of the integrable classes presented by Abel, Liouville and Appell in [2, 3, 4]. In turn the methods they used to obtain new integrable classes seem to be forgotten or not mentioned elsewhere. So, it appeared reasonable to start by reviewing and analyzing selected parts of those works in this section, and then show in the next section how, starting from these ideas, additional integrable classes can be obtained.

The first large presentation of integrable cases is due to Abel himself in [2]. His idea was to consider integrating factors of the form

$$
\mu=\mathrm{e}^{r(x, y)}
$$

for "Abel" equations written in terms of two arbitrary functions $p$ and $q$ as:

$$
\Phi \equiv y y^{\prime}+p(x)+q^{\prime}(x) y=0
$$


The first non-trivial case discussed in [2] was found by taking $r(x, y)$ as quadratic in $y$ :

$$
\mu=\mathrm{e}^{\left(\alpha+\beta y+\gamma y^{2}\right)}
$$

where $\alpha, \beta$ and $\gamma$ are arbitrary functions of $x$. Abel formulated this problem by applying Euler's operator to the total derivative $\mu \Phi$, obtaining a system easily solvable for $\alpha, \beta, \gamma$ and $p$. The resulting Abel family has non-constant invariant and is shown in Abel's memoires as depending on one arbitrary function $q(x)$ and two arbitrary constants $C_{i}$ :

$$
y y^{\prime}-\frac{q^{\prime}}{2 C_{1} q+C_{2}}+q^{\prime} y
$$

(for the corresponding integrating factor see [2]]). Now, for the purpose of building computer routines addressing the equivalence problem, it is crucial to determine whether or not a given class depends on parameters since, as explained in sec. 3, in such a case the formulation of that problem is much more difficult. In the case of Eq.(29), the two parameters $C_{i}$ and the function $q(x)$, can be removed by first converting the ODE to first kind using $y(x)=1 / v(x)$, and then employing a transformation of the form Eq.(2): $\left\{x=F(t), v(x)=u(t) \sqrt{-2 C_{1}}\right\}$, with $F$ implicitly defined by $2 C_{1} q(F)-t \sqrt{-2 C_{1}}+C_{2}=0$, arriving at a representative of the class simpler than Eq.(29),

$$
y^{\prime}=\frac{y^{3}}{x}+y^{2}
$$

and showing that this class does not depend on parameters. It is then easy to verify that Eq.(30) is a particular case of a parameterized class $[7$ derived from Appell's work [4].

The next integrable case shown by Abel is obtained by considering for Eq.(28) an integrating factor of the form $\mu=\exp (1 /(\alpha+\beta y))$. Proceeding as in the previous case, Abel arrived at another integrable ODE class with non-constant invariant, which however (see [3]) is a particular member of the parameterized class Eq.(33) shown by Abel in the same paper.

\section{Constant Invariant case}

Abel then considered an integrating factor of the form $\mu=(\alpha+\beta y)^{n}$. This ansatz does not lead to a non-constant invariant family. However, this is the first presentation we have found of a method for the constant invariant case. Liouville, and others after him, rediscovered this method, presented in Kamke as due to M. Chini [9], and in Murphy's book as a change of variables mapping an Abel ODE into a separable one. A recent discussion of the symmetries of this constant invariant problem is found in [6].

Class "A" depending on one arbitrary parameter

The next ansatz considered by Abel was

$$
\mu=(A+y)^{a}(B+y)^{b} y
$$

where $A(x)$ and $B(x)$ are arbitrary functions and $a$ and $b$ are arbitrary constants. By taking $b=-a$ Abel showed that a tractable integrable case results:

$$
y y^{\prime}+\frac{q^{\prime}}{4 q}\left(\left(q+2 \frac{C_{1}}{q}\right)^{2}-\frac{q^{2}}{a^{2}}\right)+q^{\prime} y=0
$$

The arbitrary function $q(x)$ can be removed together with the constant $C_{1}$ by rewriting this ODE in first kind format, and then appropriately choosing $\{F, P, Q\}$ in Eq.(2); so that a simpler representative of this class depending on only one parameter " $\alpha$ ", is given by 18

\footnotetext{
${ }^{17}$ Eq. (30) is obtained from Eq.(58) taking $C=0$ and changing variables $\{x=i t, y=i u(t)\}$.

${ }^{18} \mathrm{~A}$ representative of the same class of Eq. 33) is shown in [3] as $y^{\prime}=\frac{4}{9 x^{3}}\left(\left(x^{2}+1\right)^{2}-c x^{4}\right) y^{3}+\frac{4 y^{2}}{3}$
} 


$$
y^{\prime}=\left(\alpha x+\frac{1}{x}+\frac{1}{x^{3}}\right) y^{3}+y^{2}
$$

Class 1

In [10], Halphen noted a connection between doubly-periodic elliptic functions and the Abel type ODE

$$
y^{\prime}=\frac{3 y(1+y)-4 x}{x(8 y-1)}
$$

which transforms into itself under infinitely many rational changes of variables, from where he was able to determine both a parametric and an algebraic solution for it (see the Appendix).

Class 2

In a paper by Liouville [3] mostly dedicated to Abel equations, he discussed the integrable cases known at that time (1903), and presented some new ones. Liouville reviewed Abel's work and considered for Eq.(28) an integrating factor of the form Eq.(27) with $r(x, y)$ cubic in $y$, arriving at the integrable family $y^{\prime}=6 a x y^{2}+3 a y^{3}$, depending on a parameter $a$. This parameter however can be removed by changing variables as in $\{y=-u(t) / \sqrt[3]{3 a}, x=t / \sqrt[3]{3 a}\}$ arriving at the integrable class free of parameters represented by

$$
y^{\prime}=-2 y^{2} x+y^{3}
$$

Class "B" depending on one arbitrary parameter

As a generalization of Eq.(35), in [3] Liouville also presented the parameterized family

$$
y^{\prime}+\left(3 m x^{2}+4 m^{2} x+n\right) y^{3}+3 x y^{2}=0
$$

written in terms of two parameters $m$ and $n$ and which can be mapped into a Riccati ODE solvable in terms of special functions. Eq.(35) is a member of the class represented by Eq.(36) after setting $m=0$. However, when $m=0, n$ can be removed from Eq.(36) by changing variables $\left\{x=t \sqrt[3]{n}, y(x)=t u(t) / n^{2 / 3}\right\}$, leading to a class without parameters - actually represented by Eq.(35). In turn, when $m \neq 0, m$ and $n$ can be "merged" by changing variables $\left\{y=u(t) / m^{2}, x=m t\right\}$ and introducing a new parameter $a=n / m^{3}$, resulting in

$$
y^{\prime}=-\left(3 x^{2}+4 x+a\right) y^{3}-3 x y^{2}
$$

In summary, Eq.(36) is not a full 2-parameter class, but instead two classes represented by Eqs.(35) and (37), respectively depending on zero and one parameters. A simpler representative for this class and its solution are found in the Appendix.

$\underline{\text { Class } 3}$

Still in [3] Liouville pointed out that by interchanging the role between the dependent and independent variables in Eq.(35) one arrives at a different Abel integrable class. After rewriting this resulting ODE in first kind format and performing a change of variables of the form Eq.(2)), a simpler representative of this integrable class is given by

$$
y^{\prime}=\frac{y^{3}}{4 x^{2}}-y^{2}
$$




\subsection{Integrable Abel ODE classes shown in Kamke and some others books}

One of the most well known collection of (69) Abel ODEs is the one shown in Kamke's book. This collection however makes no distinction between constant or non-constant invariant cases, presents ODEs of the same class as different, and does not discuss what would be the representative for each class depending on the least number of parameters. A first classification for these Abel ODEs is then given by

\begin{tabular}{|l|l|}
\hline Classification & ODE numbers as in Kamke's book \\
\hline 4 are too general & $50,219,250,269$ \\
\hline 40 constant invariant & $38,41,46,49,51,188,204,213,214,215,216,218,221,222,223,224,225$, \\
& $226,227,228,229,231,236,238,239,243,244,245,246,247,248,249,251$, \\
& $252,254,255,260,261,262,264$ \\
\hline 24 non-constant invariant & $36,37,40,42,43,45,47,48,111,145,146,147,151,169,185,203,205,206$, \\
& $234,235,237,253,257,265$ \\
\hline 10 shown without solution & $40,47,48,203,205,206,234,237,253,265$ \\
\hline
\end{tabular}

Table 1. First classification for the 69 Abel ODEs shown in Kamke's book.

As mentioned, all constant invariant ODEs can systematically be transformed into separable ODEs (see for instance Murphy's book), so that the interesting subset is the one comprising 24 ODEs having non-constant invariants. We note also that 10 of these 24 ODEs are shown in the book without a solution, and in fact we were unable to solve any of 203, 205, 206, 234, 253 or 265, so that the number of integrable cases for us is 18 .

From these 18 ODEs (and hence from the 69 Abel type Kamke's examples), only four - those numbered: 47, 185, 235 and 237- would really lead to additional integrable classes with respect to those presented in the works by Abel, Liouville and Appell. We note however that the examples 47, 185 and 237 are all members of Class "C" (see Eq.(49)), which can be derived from the work by Abel [2] - even when it was not presented in the original work. So that the number of additional integrable classes presented in Kamke reduces to one, represented by the example 235. The classification and details are as follows.

Class 4

$$
(x y+a) y^{\prime}+b y=0
$$

This ODE (K 1.235) is presented in Kamke in terms of two arbitrary parameters $\{a, b\}$; then, a change of variables which transforms it into a linear ODE is shown. A simpler representative of this class - not depending on parameters - can be obtained by rewriting this equation in first kind format via $\left\{x=t, y=\frac{1}{t u(t)}-\frac{a}{t}\right\}$ and then changing variables $\left\{x=\frac{a}{t b}, y=\frac{t u(t)}{a}\right\}$, leading to

$$
y^{\prime}=y^{3}-\frac{(x+1)}{x} y^{2}
$$

Comments on Kamke's example 47

For the ODE

$$
y^{\prime}-a\left(x^{n}-x\right) y^{3}-y^{2}=0
$$

\footnotetext{
${ }^{19}$ In this classification, by "too general" we mean: these ODEs cannot be solved without restricting the example to a concrete particular case. We excluded ODEs - like those numbered 230 and 232 - which are already of Bernoulli type. We note also that the ODEs shown in Kamke without solution can all be transformed into an Emden type second order ODE shown in Kamke as 6.74, for which only a general discussion is presented. In turn, a detailed discussion on the integrable cases of Emden type ODEs is found in [1].
} 
presented in Kamke as K 1.47, there is no solution shown in the book, but instead a suggestion of transforming the ODE into a second order one. We followed that suggestion and then ran a symmetry analysis, noticing that the resulting ODE will have two point symmetries if either $\left\{a=-\frac{2 n+2}{9+6 n+n^{2}}\right\}$ or $\left\{n=2, a=\frac{6}{25}\right\}$, leading to two integrable classes not shown in the book. In the former case, from Eq.(41), we arrive at

$$
y^{\prime}+\frac{(2 n+2)\left(x^{n}-x\right) y^{3}}{9+n^{2}+6 n}-y^{2}=0
$$

However, this ODE can be transformed into Eq.(49) by changing variables $\left\{x=t^{\frac{2}{1-n}}, y=-u(t) \frac{n+3}{2} t^{\frac{n+1}{n-1}}\right\}$ followed by $n=\frac{a+2}{a-2}$, so that it belongs to Class C. In the same line, taking $\left\{n=2, a=\frac{6}{25}\right\}$ in Eq.(41), and changing variables $\left\{x=\frac{t^{2}-1}{t^{2}}, y=5 / 2 u(t) t^{3}\right\}$ one arrives at Eq.(49) with $a=6$, so that this second branch of Eq.(41) is also a member of Class C.

Comments on Kamke's example 237

$$
x(y+a) y^{\prime}+b y+c x=0
$$

This ODE (K 1.237) depending on three arbitrary parameters $\{a, b, c\}$, is presented in the book without a solution. We note however that changing $\{x \rightarrow y, y \rightarrow x\}$ leads to an ODE also of Abel type and in second kind format. Converting the latter to first kind format via $\{x=t, y=$ $\left.\frac{1}{c u(t)}-\frac{b t}{c}\right\}$, replacing $y \rightarrow y^{\prime}$ and running a symmetry analysis, the resulting second order ODE has two symmetries when $a=-2 b$, leading to an integrable case. Introducing $a=-2 b$ into Eq.(43), rewriting it in first kind format via $\left\{x=t, y=-\frac{1}{t u(t)}+2 b\right\}$ and changing variables $\left\{x=-\frac{b^{2}(t+4)}{2 c}, y=\frac{2 c u(t)}{b^{3}(t+4)}\right\}$ leads to a simpler representative of the class not depending on any parameters:

$$
y^{\prime}=\frac{-x y^{3}+2 y^{2}}{2(x+4)}
$$

However, by changing variables $\left\{x=4\left(1-t^{2}\right) / t^{2}, y=-u(t) t / 2\right\}$ one arrives at Eq.(49) again, this time with $a=-1 / 2$, so that Eq.(44) is also member of Class C.

A classification for all these 18 non-constant invariant Kamke examples is then as follows20

\begin{tabular}{|c|c|c|c|c|c|c|}
\hline Class 2 & Class 3 & Class 4 & Class A & Class B & Class C & Class D \\
\hline 36,40 & 145,147 & 235 & 257 & 42,43 & $45,47,48,151,185,237$ & $37,111,146,169$ \\
\hline
\end{tabular}

Table 2. Classification for the 18 non-constant invariant solvable Abel ODEs in Kamke's book.

where classes $\mathrm{C}$ and $\mathrm{D}$ are defined in sec. 5. In summary, all but one of Kamke's 58 solvable examples (18 non-constant invariant +40 constant invariant) are particular cases of the integrable classes presented by Abel, Liouville and Appell in [2, 3, 4], or can be derived from there (those belonging to Classes $\mathrm{C}$ and $\mathrm{D}$ ).

Another collection of Abel ODEs is found in the book by Murphy [5]. After selecting those examples not having a constant invariant and for which a solution is shown in the book, we arrived at a set of nine ODEs, numbered in the book as: 78, 79, 80, 86, 275, 304, 345, 383 and 593. None of these ODEs represent an additional integrable class; their distribution among the classes discussed in this work is as follows

\footnotetext{
${ }^{20}$ Equations K.1.47, K.1.48 and K.1.237 belong to Class C for infinitely many - however particular - values of one of the two parameters (see Eq.(42)); we don't know their solution for other values.
} 


\begin{tabular}{|c|c|c|c|c|}
\hline Class 2 & Class 3 & Class B & Class C & Class D \\
\hline 78,80 & 275 & 86 & $304,383,593$ & 79,345 \\
\hline
\end{tabular}

A wider collection of Abel ODEs than the one shown in Kamke's book is found in the book by Polyanin and Zaitsev [11]. This book is rather new (1995) and covers a vast number of integrable ODE problems which we have not found in other books, hence making the examples attractive. On the other hand the Abel ODEs shown there are classified not according to their invariants but according to their form, and the origin of their solutions is not given. Apart from a main section consisting of four tables (82 Abel ODEs - all derived from four basic ones), the book contains other sections illustrating mappings between Abel and higher order ODEs. The quantity of examples is large and the computational routines we prepared for the equivalence problem are not yet covering in full the case in which the parameters of the class may assume symbolic values. As a result we still don't have a way to solve the equivalence problem for the whole set of integrable classes presented in [11]. Our analysis of these Abel ODEs of [11] is then still incomplete; consequently we restricted the presentation here to just a sample, constituted by the ODEs of the first of these four tables. These are 20 ODEs obtained from

$$
y y^{\prime}-y=s x+A x^{m}
$$

by giving particular values to the parameters $m$ and $s$ ( $A$ is kept arbitrary). These ODEs appear in section 1.3 .1 of [11] under the numbers: $1,2,10,16,19,22,23,26,27,30,32,33,45,46,47$, $48,53,54,55$ and 56 . We were not able to classify those numbered 27, 20, 48, 55 and 56 . The distribution of the remaining ODEs, in the classes discussed in this work, is as follows:

\begin{tabular}{|c|c|c|c|c|c|}
\hline Constant invariant & Class 1 & Class 2 & Class 3 & Class C & Class D \\
\hline $1,2,26$ & 23 & 32 & 33 & $10,19,22,45,46,47,53,54$ & 16 \\
\hline
\end{tabular}

\section{$5 \quad$ New integrable Abel ODE classes derived from previous works}

Class "C" depending on one arbitrary parameter

The form of the integrating factor studied by Abel actually leads to other integrable cases not mentioned in the original work [2]. One of them is obtained by taking $b=a$ in Eq.(31), resulting in the ODE family巴

$$
y y^{\prime}-q^{\prime} y-\frac{q^{\prime} n^{2}\left(-\frac{q}{n}+C_{1}^{2}\left(\frac{q}{n}\right)^{2 n-1}\right)}{(n+1)^{2}}=0
$$

where $n \neq-1$. The function $q(x)$ and the parameter $C_{1}$ can be removed as done with Eq.(32), leading to

$$
y^{\prime}=n\left(x-x^{2 n-1}\right) y^{3}-(n+1) y^{2}
$$

which is turned exact by means of the integrating factor

$$
\mu=\frac{\left(1+\left(\left(x^{2}-x^{2 n}\right) y-2 x\right) y\right)^{-\frac{n+1}{2 n}}}{y^{\frac{2 n-1}{n}}}
$$

\footnotetext{
${ }^{21} n$ in Eq.(46) is related to $a$ in Eq.(31) by $n=-1 /(2 a+1)$
} 
A simpler representative of this class is obtained by changing variables $\left\{y=u(t) t^{\frac{n}{n-1}}, x=t^{\frac{1}{1-n}}\right\}$, then introducing a new parameter by means of $n=\frac{\alpha}{\alpha-2}$, arriving at

$$
y^{\prime}=\frac{\alpha\left(1-x^{2}\right) y^{3}}{2 x}+(\alpha-1) y^{2}-\frac{\alpha y}{2 x}
$$

Taking into account Eq.(48), an implicit solution for this class is given by

$$
C_{1}+\frac{\alpha}{x}\left(1-\frac{(1-x y)^{2}}{y^{2}}\right)^{1 / \alpha}-2 \int^{\frac{1-x y}{y}}\left(1-z^{2}\right)^{\frac{1-\alpha}{\alpha}} d z=0
$$

Class " $D$ " depending on one arbitrary parameter

In [4], Appell showed a series of integrable cases derived from the solutions to

$$
u^{\prime}=A(u)+B(u) t
$$

By changing variables $\left\{t=\frac{1}{y}-\frac{A(x)}{B(x)}, u=x\right\}$, this ODE is transformed into the Abel ODE

$$
y^{\prime}=-\frac{y^{3}}{B}-\left(\frac{A}{B}\right)^{\prime} y^{2}
$$

where $A$ and $B$ are now functions of $x$. Any particular $\{A, B\}$ leading to a solvable case in Eq.(51) will then also lead to an integrable Abel ODE Eq. (52). Among the choices for $\{A, B\}$ considered in [1] - such that Eq.(51) results linear, homogeneous, or of Riccati type - only this mapping into Riccati type leads to something new. This case is obtained by taking

$$
A=a x^{2}+b x+c, \quad B=\alpha x^{2}+\beta x+\gamma
$$

The related Abel ODE family, depending on six parameters $\{a, b, c, \alpha, \beta, \gamma\}$, is given by

$$
y^{\prime}=-\frac{y^{3}}{\alpha x^{2}+\beta x+\gamma}-y^{2} \frac{d}{d x}\left(\frac{a x^{2}+b x+c}{\alpha x^{2}+\beta x+\gamma}\right)
$$

and its solution could be expressed in terms of the solution to the Riccati ODE

$$
y^{\prime}=(a+\alpha x) y^{2}+(b+\beta x) y+c+\gamma x
$$

However, we were not able to solve this Riccati ODE for arbitrary values of the six parameters involved and in [4] there is no indication of how that could be done. The alternative we then investigated is to consider the second order ODE obtained by replacing $y=y^{\prime}$ in Eq.(55). That ODE has two point symmetries if and only if $\alpha=0$. With these symmetries we were able to solve that second order ODE, and hence Eq.(55) when $\alpha=0$. Concerning the related Abel family Eq.(54) - now depending on five parameters - an appropriate change of variables of the form Eq.(2)

$$
\left\{x=\frac{t \sqrt{\beta}}{a}-\frac{\gamma}{\beta}, \quad y=\sqrt{\beta} u(t)\right\}
$$

followed by the introduction of a new parameter $C$ by means of

$$
C=-\frac{\left(\beta^{2} c+\alpha \gamma^{2}\right) a-\alpha \beta \gamma b}{\beta^{2}}
$$


transforms Eq.(54) into a simpler representative for the class

$$
y^{\prime}=-\frac{y^{3}}{x}-\frac{\left(C+x^{2}\right) y^{2}}{x^{2}}
$$

also showing that this class depends not on five but on one parameter 2 . It appeared of value to us also to determine the number of parameters on which Eq.(54) depends in the general case, that is before taking $\alpha=0$. For that purpose we searched for the appropriate changes of variables of the form Eq.(2) which would remove as many as possible of these parameters, requiring that both the change and its inverse are finite. We then considered the branches which become infinite for some particular values of the parameters $\{a, b, c, \alpha, \beta, \gamma\}$ entering the transformations found. The results are summarized as follows. If all these parameters are different from zero, introducing new parameters $\{A, B, C, G\}$ by means of

$$
\begin{aligned}
\alpha & =\frac{\beta^{2}+4 A^{4}}{4 \gamma} \\
b & =\frac{8 \beta \gamma^{2} a A^{2} B+C}{2 \gamma A^{2} B\left(\beta^{2}+4 A^{4}\right)} \\
c & =\frac{A^{2} B C+16 \gamma^{2} a A^{6} B+\beta C+4 \beta^{2} \gamma^{2} a A^{2} B}{A^{2} B\left(\beta^{2}+4 A^{4}\right)^{2}} \\
\gamma & =\frac{C}{2 A^{3} B G\left(\beta^{2}+4 A^{4}\right)}
\end{aligned}
$$

followed by changing variables $\left\{x=\frac{C\left(2 t A^{2}-\beta\right)}{\left(\beta^{2}+4 A^{4}\right)^{2} A^{3} B G}, y=u(t) A\right\}$ in the six-parameter Eq. (54), one arrives at a 2-parameter representative for the same class

$$
y^{\prime}=-\frac{y^{3}}{x^{2}+1}+\frac{G\left(B x+x^{2}-1\right) y^{2}}{\left(x^{2}+1\right)^{2}}
$$

Now the case $\alpha=0$ was already shown to lead to Eq.(58), and all the other possible branches obtained from Eq.(54) by taking some of the other parameters equal to zero lead either to constant invariant families, or to members of the classes already discussed in this work 23

Three new classes not depending on parameters

While analyzing the works [2, 3, 4] and Kamke's examples, a large number of symbolic experiments were performed, sometimes leading to intermediate results which with a bit more work appeared to be new integrable classes by themselves. This happened three times, resulting in classes 5,6 and 7, for which representatives and solutions are given as follows:

Class 5

$$
y^{\prime}=-\frac{(2 x+3)(x+1) y^{3}}{2 x^{5}}+\frac{(5 x+8) y^{2}}{2 x^{3}}
$$

\footnotetext{
${ }^{22}$ We note that in this process we have made two implicit assumptions: $a \neq 0$ and $\beta \neq 0$. To assure that the cases in Eq. 54 are covered by Eq. 58) we then also considered $a=0$ and $\beta=0$ separately, arriving at ODEs respectively members of the classes represented by Eq.(58) and Eq.(35).

${ }^{23}$ There is a special case, when $b=4 \frac{\gamma \beta a}{\beta^{2}+4 A^{4}}$, where the resulting Abel ODE can only be obtained from Eq.(60) by taking appropriate limits.
} 
Solution:

$$
C_{1}+\frac{\sqrt{A}}{\sqrt[4]{4 \frac{(x+1)^{2}}{x^{2} A}+1}}+\int^{2 \frac{x+1}{x \sqrt{A}}}\left(z^{2}+1\right)^{-5 / 4} d z=0
$$

where $A=\frac{4}{y}-\frac{10}{x}-\frac{6}{x^{2}}-4$.

Class 6

$$
y^{\prime}=-\frac{y^{3}}{x^{2}(x-1)^{2}}+\frac{\left(1-x-x^{2}\right) y^{2}}{x^{2}(x-1)^{2}}
$$

Solution:

$$
C_{1}-\operatorname{Ei}\left(1, \frac{y+x^{2}-x}{x y(x-1)}\right)+\frac{(x-1) y \mathrm{e}^{\frac{x-y-x^{2}}{x y(x-1)}}}{x-1+y}=0
$$

where $\operatorname{Ei}(n, x)=\int_{1}^{\infty} \frac{\mathrm{e}^{-x t}}{t^{n}} d t$ is the exponential integral.

Class 7

$$
y^{\prime}=\frac{\left(4 x^{4}+5 x^{2}+1\right) y^{3}}{2 x^{3}}+y^{2}+\frac{\left(1-4 x^{2}\right) y}{2 x\left(x^{2}+1\right)}
$$

Solution:

$$
C_{1}+2 \frac{x+A}{\sqrt[4]{A^{2}+1}(A x-1)}+\int^{A}\left(z^{2}+1\right)^{-5 / 4} d z=0
$$

where $A=\frac{x-2 y x^{4}-3 y x^{2}-y}{x\left(x+y x^{2}+y\right)}$

\section{Computer algebra routines, tests and performance}

The two itemized algorithms described in sections 2.2 and 3 for solving the equivalence problem between two given Abel ODEs were implemented in Maple R5, in the framework of the ODEtools package [12]. The implementation consists of various routines, mainly accomplishing the following:

1. determine whether a given Abel ODE belongs to one of the solvable classes described in the previous sections; in doing that, determine also the function $F(t)$ entering Eq.(2) and the value of the parameters in the case of a parameterized class;

2. use that information to determine the functions $P(t)$ and $Q(t)$ entering Eq.(2) and return a solution to the given ODE by means of changing variables in the solution available for the representative of the class (see the Appendix).

\subsection{Representatives with simpler invariants for Classes A, C and D}

While preparing the computational routines being presented, we noticed that: if on the one hand the solutions to the representatives for the parameterized classes A, C and D (Eqs.(33, 49, 58)) can be expressed in a relatively simple manner (see the Appendix), on the other hand, for each of these representatives, the form of the three invariants entering Eq.(22) is much simpler if an appropriate redefinition of the class parameter followed by a change of variables of the form Eq.(2) is performed. In turn, the complexity of these invariants is a relevant issue for a computer algebra implementation since simpler invariants lead to simpler GCD and resultant computations. 
In the case of Class A (Eq.(33)), redefining the parameter as $\alpha=5 / 36-\kappa / 3$ - where $\kappa$ is the new parameter - and changing variables $\left\{x=\frac{\sqrt{12 t-6}}{2 t-1}, y=\frac{(2 t-1) u(t)}{\sqrt{12 t-6}}\right\}$ lead to the class representative

$$
y^{\prime}=\frac{\left(\left(3 \kappa-2 x-x^{2}\right) y^{3}-9 y^{2}-9 y\right)}{9(2 x-1)}
$$

and the first invariant $s_{5}^{3} / s_{3}^{5}$ for it is

$$
\frac{\left(9 \kappa^{2}+\left(30 x^{2}-6 x+3\right) \kappa-6 x^{3}+5 x^{4}+7 x^{2}\right)^{3}}{9\left(\kappa+x^{2}\right)^{5}}
$$

as opposed to

$$
729 \frac{\left(27 \alpha^{2} x^{8}-\left(72 x^{6}+270 x^{4}+15 x^{8}\right) \alpha+54 x^{4}+36 x^{2}+135+15 x^{6}+2 x^{8}\right)^{3}}{x^{4}\left(2 x^{4}-9 \alpha x^{4}+9 x^{2}+27\right)^{5}}
$$

which is the same first invariant but calculated for Eq.(33). Actually a measure of the Maple computational length of the three invariants $\left[s_{5}^{3} / s_{3}^{5}, s_{3} s_{7} / s_{5}^{2}, s_{5} s_{7} / s_{3}^{4}\right]$ entering Eq. (22) shows the values [99, 215, 223] when calculated on Eq.(67) above and [146, 354, 356] when calculated on Eq.(33). Also, the degrees in $x$ of both numerator and denominator of Eq.(68) are lower than the corresponding degrees of Eq.(69).

A similar situation happens with Class C (Eq. 49$)$ ), where redefining the parameter as $\alpha=\frac{\kappa-3}{\kappa}$ and changing variables $\left\{x=\frac{\sqrt{(\kappa+1)(3-\kappa)(3 t+4) t}}{(\kappa+1) t}, y=\frac{t^{2} u(t) \kappa((3 t+4) \kappa+3 t+4)}{\sqrt{(\kappa+1)(3-\kappa)(3 t+4) t}}\right\}$ lead to a class representative a bit more complicated than Eq.(49):

$$
y^{\prime}=4((\kappa-2) x+\kappa-3) \kappa x y^{3}+6 y^{2}-\frac{(6 \kappa x+5 \kappa+3) y}{(3 x+4) \kappa x}
$$

for which, however, the computational length of the three invariants $\left[s_{5}^{3} / s_{3}^{5}, s_{3} s_{7} / s_{5}^{2}, s_{5} s_{7} / s_{3}^{4}\right]$ is $[110,273,305]$ as opposed to $[173,382,412]$ when calculated for Eq.(49). The degrees with respect to $x$ of the numerators and denominators of the invariants of Eq.(70) are also lower than those of the invariants of Eq.(49).

The same reduction in the complexity of the invariants is achieved for class D (Eq.(58)) by redefining the parameter via $C=\frac{9}{8} \sqrt{\kappa}$ and changing variables $\left\{x=\frac{3 \sqrt{2\left(1-t^{2} \kappa\right) \sqrt{\kappa}}}{4(1-t \sqrt{\kappa})}, y=\sqrt{2\left(1-t^{2} \kappa\right) \sqrt{\kappa}} u(t)\right\}$, leading to

$$
y^{\prime}=\frac{\left(2 x^{2} \kappa-2\right) \kappa y^{3}-3 y^{2} \kappa+\kappa x y}{1-\kappa x^{2}}
$$

for which the first invariant, $s_{5}^{3} / s_{3}^{5}$, is given by

$$
\frac{\left(\left(2 x^{4}+15 x^{3}\right) \kappa^{2}-\left(4 x^{2}+15 x-9\right) \kappa+2\right)^{3}}{4 \kappa^{3}\left(\kappa x^{3}-x+1\right)^{5}}
$$

as opposed to

$$
729 \frac{\left(C+x^{2}\right)^{3}\left(2 C^{4}+8 x^{2} C^{3}+\left(12 x^{4}+15 x^{2}\right) C^{2}+8 x^{6} C+2 x^{8}-15 x^{6}+9 x^{4}\right)^{3}}{\left(2 C^{3}+6 C^{2} x^{2}+\left(9 x^{2}+6 x^{4}\right) C+2 x^{6}-9 x^{4}\right)^{5}}
$$

\footnotetext{
${ }^{24}$ The case $\kappa=0$ is treated separately.
} 
which is the same first invariant but calculated for the class representative Eq.(58). In Eq.(72), not only the degrees with respect to $x$ but also those with respect to the class parameter $\kappa$ are lower than the corresponding degrees in Eq.(73).

Moreover, for class D this change in the representative of the class also fixes a problem: the representative Eq.(58) is itself invariant under $C \rightarrow-C$ followed by the change of variables

$$
\left\{x=\frac{i C}{t}, y=i u(t)\right\}
$$

where $i$ is the imaginary unit, and hence Eq.(73) is invariant under $\{C \rightarrow-C, x \rightarrow i C / x\}$. Consequently, if $\{C, F(t), P(t), Q(t)\}$ is a solution to the equivalence problem between a given ODE and Eq.(58), then $\left\{-C, \frac{i C}{F(t)}, i P(t), Q(t)\right\}$ is also a solution; this fact invalidates the use of the interpolation method described in sec. 3.2 with Eq.(58) since that method can only be used when the interpolated solution $C(\alpha)$ is unique.

\subsection{Installation}

The programs being presented have been written as one more step in the development of the ODEtools Maple package [12] and hence are integrated to it and not distributed separately. To install the new Abel routines then what is necessary is to install ODEtools to run in the Maple environment by putting the related libraries (two files - maple.ind and maple.lib) in any directory - say DE_libraries_directory - then opening Maple, and adding that directory to Maple's libname variable via

$>$ libname := DE_libraries_directory, libname:

where ' $>$ ' is the Maple prompt. This instruction automatically updates Maple's dsolve subroutines to make use of the new routines for solving Abel ODEs - no further steps are required. In this way, the new Abel ODE routines are automatically used by Maple's dsolve when the input ODE is of Abel type, as well as to solve higher order ODEs when they can be reduced to first order Abel ODEs members of the classes discussed in sec. 1 and 5 .

Apart from this integration with dsolve, it is also possible to try just the routines being presented by giving to dsolve the extra argument [Abel]. For example (Kamke's ODE 37)

$>\operatorname{ode}[37]:=\operatorname{diff}(y(x), x)-y(x) \wedge 3-a * \exp (x) * y(x)^{\wedge} 2=0$;

$$
\text { ode }_{37}:=y^{\prime}-y^{3}-a \mathrm{e}^{x} y^{2}=0
$$

$>$ dsolve(ode [37], [Abel]);

$$
C_{1}+\frac{\mathrm{e}^{-1 / 2\left(a \mathrm{e}^{x}+y^{-1}\right)^{2}}}{a \mathrm{e}^{x}}+\frac{\sqrt{2 \pi}}{2} \operatorname{erf}\left(\frac{\sqrt{2}}{2}\left(a \mathrm{e}^{x}+y^{-1}\right)\right)=0
$$

These implicit answers can also be tested in the Maple worksheet, interactively, using the standard Maple odetest command.

Due to the intrinsic complexity of Abel equations, the solution for most of the solvable classes is expressed in implicit form and in terms of elliptic integrals and special or hypergeometric functions. Then, to save the time Maple spends in trying to "integrate" these integrals or to "invert" these algebraic expressions it is frequently convenient to call dsolve with the optional extra arguments 
'useInt, implicit', meaning: use Maple's inert Int and return the solution directly in implicit form²5. For example, for Kamke's 185

$>\operatorname{ode}[185]:=x^{\wedge} 7 * \operatorname{diff}(y(x), x)+2 *\left(x^{\wedge} 2+1\right) * y(x)^{\wedge} 3+5 * x^{\wedge} 3 * y(x)^{\wedge} 2=0$

$$
\text { ode }_{185}:=x^{7} y^{\prime}+2\left(x^{2}+1\right) y^{3}+5 x^{3} y^{2}=0
$$

the solution obtained using these extra arguments is:

$>$ dsolve(ode[185], [Abel], useInt, implicit);

$$
C_{1}+\frac{x}{\sqrt[4]{A^{2}+1}}+1 / 2 \int^{A}\left(z^{2}+1\right)^{-5 / 4} d z=0
$$

where $A=x^{-1}+\frac{x^{2}}{y}$. The explicit computation of the integral above leads to a complicated expression with hypergeometric functions somehow obscuring the structure of the solution.

Concerning Maple's difficulty in solving the problem when the class parameter $\mathcal{C}$ involves radicals (see end of sec. 3.1), we implemented an environment variable controlling how hard the routines will work, in order to avoid exhausting system resources unless a hard trial is specifically requested. This environment variable is _Env_odsolve_Abel_try_hard, it can be assigned a positive integer from 1 to 5, and by default it is assigned to 4; the meaning of the possible values is as follows:

- if _Env_odsolve_Abel_try_hard = 1 then the algorithm for parameterized Abel classes is disabled, and for non-parameterized classes only a restricted equivalence using $\{x=t, y=$ $P(t) u+Q\}$, that is: with $F(t)=t$ in Eq.(2), is tried;

- if _Env_odsolve_Abel_try_hard $=2$ then for non-parameterized classes a full equivalence using Eq.(2) is tried;

- if _Env_odsolve_Abel_try_hard $=3$ then the algorithm for parameterized Abel classes is enabled but only for numerical solutions for the class parameter $\mathcal{C}$;

- if _Env_odsolve_Abel_try_hard = 4 then for parameterized Abel classes both a numerical or a rational interpolation solution for the class parameter $\mathcal{C}$ are tried (symbolic variables in the coefficients are allowed but solutions with radicals are not computed) ;

- if _Env_odsolve_Abel_try_hard = 5 then the algorithm for parameterized Abel classes will also compute solutions involving radicals for the class parameter $\mathcal{C}$.

Finally, these routines for Abel ODEs were programmed to provide extensive run-time information on the computations being performed through the Maple standard userinfo \& infolevel scheme. For example, Kamke's ODE 43 belongs to class B (see Table 2.) and both the way how this is determined and the explicit values found for $F, P, Q$, and the parameter entering Eq.(37) can be seen by setting the infolevel as follows ${ }^{20}$ :

$>$ infolevel[dsolve] := 4;

$>$ ode [43] :=

\footnotetext{
${ }^{25}$ The option implicit is standard in Maple's dsolve and the implementation of the option useInt comes with the routines for Abel ODEs presented here.

${ }^{26}$ We kept few lines to illustrate the userinfo feature, and represented the missing ones by '....'.
} 


$$
\text { ode }_{43}:=y^{\prime}+\left(3 a x^{2}+4 a^{2} x+b\right) y^{3}+3 y^{2} x=0
$$

$>$ dsolve(ode [43], [Abel], implicit);

$$
\text { .... }
$$

The relative invariant s3 is: $-3 * a * x^{\wedge} 2+b-2 * x^{\wedge} 3$

The first absolute invariant s5^3/s3^5 is: $108 *\left(12 * a \wedge 2 * x^{\wedge} 3-8 * a * x * b+\ldots\right.$

The second absolute invariant $\mathrm{s} 3 * \mathrm{~s} 7 / \mathrm{s} 55^{\wedge} 2$ is: $1 / 3 *\left(3 * a * x^{\wedge} 2-b+2 * x^{\wedge} 3\right) * \ldots$

The third absolute invariant s5*s7/s3^4 is: $9 *\left(372 * a^{\wedge} 3 * x^{\wedge} 4+450 * a \wedge 2 * x^{\wedge} 5+\ldots\right.$

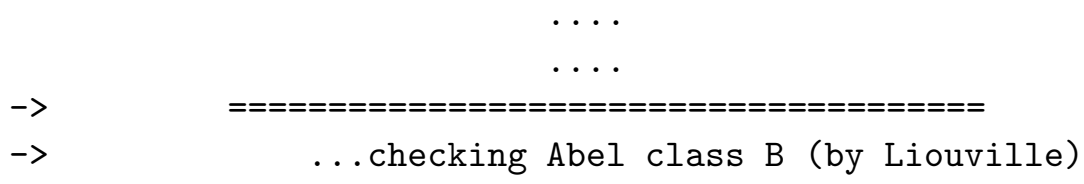

Trying $a=0$

Trying $a=1$

Trying $\mathrm{b}=0$

Trying $b=1$

-> Step 1: checking for a disqualifying factor on $F$ after evaluating $x$

Trying $\mathrm{x}=0$

*** No disqualifying factor on $\mathrm{F}$ was found $* * *$

$\rightarrow$ Step 2: calculating resultants to eliminate $\mathrm{F}$ and get candidates for $\mathrm{C}$

*** Candidates for $\mathrm{C}$ are $[1,4,1 / 4]$, ***

-> Step 3: looking for a solution $\mathrm{F}$ depending on $\mathrm{x}$

$\mathrm{C}=1 / 4$ leads to the solutions $[\{\mathrm{F}=-1-3 / 2 * \mathrm{x}\}]$

Interpolated candidate for the class parameter $\mathrm{C}$ is: $\mathrm{C}=1 / 4$

General testing of the candidate $C=1 / 4$ with arbitrary $b$

Interpolation is still incomplete; trying next value of $b$

Trying $\mathrm{b}=2$

$\cdots$

...

General testing of the candidate $C=-3 / 4 * b+1$ with arbitrary $b$

$\mathrm{C}=-3 / 4 * \mathrm{~b}+1$ leads to the solutions $[\{\mathrm{F}=-1-3 / 2 * \mathrm{x}\}]$

General test of $\mathrm{C}=-3 / 4 * b+1$ passed $O K$;

interpolation for $b$ in this level is complete.

....

$\ldots$

General testing of the candidate $\mathrm{C}=(-3 / 4 * \mathrm{~b}+\mathrm{a} \wedge 3) / \mathrm{a} \wedge 3$ with arbitrary $\mathrm{a}$

$\mathrm{C}=\left(-3 / 4 * \mathrm{~b}+\mathrm{a}^{\wedge} 3\right) / \mathrm{a}^{\wedge} 3$ leads to the solutions $[\{\mathrm{F}=-1 / 2 *(3 * \mathrm{x}+2 * \mathrm{a}) / \mathrm{a}\}]$

General test of $\mathrm{C}=\left(-3 / 4 * \mathrm{~b}+\mathrm{a}^{\wedge} 3\right) / \mathrm{a} \wedge 3$ passed $\mathrm{OK}$;

interpolation for $b$ in this level is complete.

Value of the Class parameter solving the problem is:

$\mathrm{C}=1 / 4 *\left(-3 * \mathrm{~b}+4 * \mathrm{a}^{\wedge} 3\right) / \mathrm{a}^{\wedge} 3$

Inverse of the transformation solving the problem is: 


$$
\left\{t=-1 / 2 *(3 * x+2 * a) / a, u(t)=-2 / 3 * a^{\wedge} 2 * y(x)\right\}
$$

Solution:

$$
C_{1}+\frac{\left(\left(\frac{2 a+3 x}{2 a}-A_{1}\right) \mathrm{K}\left(A_{1},-\sqrt{A_{2}}\right)-\sqrt{A_{2}} \mathrm{~K}\left(A_{1}+1,-\sqrt{A_{2}}\right)\right)}{\left(\left(\frac{2 a+3 x}{2 a}-A_{1}\right) \mathrm{I}\left(A_{1},-\sqrt{A_{2}}\right)+\sqrt{A_{2}} \mathrm{I}\left(A_{1}+1,-\sqrt{A_{2}}\right)\right)}=0
$$

where $A_{1}=\frac{1}{2} \sqrt{\frac{-3 b+4 a^{3}}{a^{3}}}, A_{2}=\frac{3 b-4 a^{3}}{4 a^{3}}+\frac{(2 a+3 x)^{2}}{4 a^{2}}-\frac{3}{2 a^{2} y}$, and I and $\mathrm{K}$ are respectively the modified Bessel functions of first and second kind.

\subsection{Tests and Performance}

The idea was to test these computational routines to confirm the correctness of the returned solutions as well as to obtain the classification presented in the previous sections for solvable Abel ODEs. The first testing arena was the 69 Abel examples found in Kamke plus the 9 solvable examples with non-constant invariant from Murphy's book, plus the first 20 Abel ODE examples from [11] mentioned at the end of sec. 4- totaling 98 Abel ODE examples. The routines passed these tests - the solutions obtained were confirmed to be correct using other symbolic computation tools interactively - and the resulting classification is that shown in Tables 1, 2, 3 and 4 of sec. 1 .

The aforementioned test however involves only 40 "non-constant invariant and solvable" Abel ODE examples, and does not fully test the new routines. We have then set up a more thorough test, which can be taken as a test-suite for other computer algebra implementations of methods for solving Abel ODEs. The ideas behind this additional test-suite are summarized as follows:

- Take the representatives of the seven Abel class not depending on parameters, discussed in sec. 1 and 5, and generate with each four more Abel ODEs of the same class by applying the different types of transformations:

1. The general case with $F, P$ and $Q$ arbitrary:

$$
\operatorname{tr}_{1}:=\{x=F(t), \quad y(x)=P(t) u(t)+Q(t)\}
$$

2. Rational transformation with symbolic coefficients $a$ and $b$ :

$$
t r_{2}:=\left\{x=\frac{a}{t}+b t, \quad y(x)=\frac{u(t)}{t}+1\right\}
$$

3. Non-rational transformation involving an elementary function and symbols:

$$
\operatorname{tr}_{3}:=\left\{x=\mathrm{e}^{t}+1+\frac{t}{a}, \quad y(x)=u(t)+1\right\}
$$

4. Non-rational transformation involving abstract powers and symbols:

$$
t r_{4}:=\left\{x=\frac{a}{t^{n}}+\frac{t}{b}, \quad y(x)=u(t)+1\right\}
$$

For example, the first of these four Abel ODEs generated from Eq.(34) is given by: 


$$
\begin{aligned}
y^{\prime}= & \frac{F^{\prime} P^{2}(-3+F) y^{3}}{8 F}+\frac{((3 F-9) Q-10) P F^{\prime} y^{2}}{8 F} \\
& +\left(\frac{\left((3 F-9) Q^{2}-20 Q-3\right) F^{\prime}}{8 F}-\frac{P^{\prime}}{P}\right) y+\frac{\left((F-3) Q^{3}-10 Q^{2}-3 Q\right) F^{\prime}}{8 F P}-\frac{Q^{\prime}}{P}
\end{aligned}
$$

and the other three are obtained from this one by replacing $F, P$ and $Q$ by the values implied by Eqs. (79), (80) and (81). The scheme just outlined generates 28 more solvable Abel ODE examples with non-constant invariant (4 per class), and suffices for testing the solving of classes without parameters.

Concerning classes with parameters:

- Take the representatives of the four Abel classes depending on parameters discussed in sec. 4 and 6.1, generate with each one four more Abel ODEs of the same class by applying the transformations Eq.(79) and Eq.(81), preceded by replacing the single parameter $\mathcal{C}$ entering each class representative by $\mathcal{C}=2$ and $\mathcal{C}=\alpha / \beta$.

This increases by $4 \times 2 \times 2=16$ more ODE examples, and the solving of these examples tests both the scheme for numeric values of the parameter $\mathcal{C}$ as well as the case where $\mathcal{C}$ is a rational function of other symbols entering the given ODE.

The time spent by the routines being presented in solving all these $28+16=44$ additional Abel ODEs with non-constant invariant, is summarized in Tables 5, 6 and 7 as follows:

\begin{tabular}{|c|c|c|c|c|}
\hline Class & transf. (i) & transf. (ii) & transf. (iii) & transf. (iv) \\
\hline 1; Eq.(34) & $0.255 \mathrm{sec}$. & $0.879 \mathrm{sec}$. & $0.687 \mathrm{sec}$. & $1.256 \mathrm{sec}$. \\
\hline 2; Eq.(35) & $1.530 \mathrm{sec}$. & $1.900 \mathrm{sec}$. & $1.998 \mathrm{sec}$. & $2.620 \mathrm{sec}$. \\
\hline 3; Eq.(38) & $0.277 \mathrm{sec}$. & $6.760 \mathrm{sec}$. & $5.623 \mathrm{sec}$. & $11.635 \mathrm{sec}$. \\
\hline 4; Eq.(40) & $0.456 \mathrm{sec}$. & $7.126 \mathrm{sec}$. & $3.941 \mathrm{sec}$. & $15.511 \mathrm{sec}$. \\
\hline 5; Eq.(61) & $0.610 \mathrm{sec}$. & $2.505 \mathrm{sec}$. & $3.082 \mathrm{sec}$. & $7.877 \mathrm{sec}$. \\
\hline 6; Eq.(63) & $1.379 \mathrm{sec}$. & $120.952 \mathrm{sec}$. & $45.565 \mathrm{sec}$. & $282.103 \mathrm{sec}$. \\
\hline 7; Eq.(65) & $0.896 \mathrm{sec}$. & $15.441 \mathrm{sec}$. & $24.586 \mathrm{sec}$. & $183.557 \mathrm{sec}$. \\
\hline
\end{tabular}

Table 5. Timings for 28 Abel ODEs with non-constant invariant - 7 classes free of parameters.

\begin{tabular}{|c|c|c|}
\hline \multicolumn{3}{|c|}{ Class parameter $\mathcal{C}=2$} \\
\hline Class & transf. (ii) & transf. (iv) \\
\hline A; Eq.(67) & $25.546 \mathrm{sec}$. & $41.304 \mathrm{sec}$. \\
\hline B; Eq.(37) & $29.396 \mathrm{sec}$. & $54.171 \mathrm{sec}$. \\
\hline C; Eq.(70) & $18.648 \mathrm{sec}$. & $41.425 \mathrm{sec}$. \\
\hline D; Eq.(71) & 36.641 sec. & $75.962 \mathrm{sec}$. \\
\hline
\end{tabular}

\begin{tabular}{|c|c|c|}
\hline \multicolumn{3}{|c|}{ Class parameter $\mathcal{C}=\frac{\alpha}{\beta}$} \\
\hline Class & transf. (ii) & transf. (iv) \\
\hline A; Eq.(67) & 67.076 sec. & 156.425 sec. \\
\hline B; Eq.(37) & 174.751 sec. & 491.210 sec. \\
\hline C; Eq.(70) & 73.940 sec. & 174.761 sec. \\
\hline D; Eq.(71) & 88.365 sec. & 177.314 sec. \\
\hline
\end{tabular}

Table 7. Timings for 8 Abel ODEs with non-constant invariant - "symbolic" class parameter.

Concerning a comparison of performances between the new routines and those available in other computer algebra systems (CAS), this appeared to us not justified in this case: roughly speaking 
none of these CAS return solutions for Abel ODEs with non-constant invariant. More precisely, from Mathematica 3.0, Macsyma 2.7, Maple 5.1 and Mupad 4.0, all of them failed 27 in solving any of the 18 Kamke's examples shown in Table 2. (and hence in solving any of the 44 ODEs of Tables 5, 6 and 7), except for Kamke's example 235 - it is an inverse linear ODE - and anyway none of them solved it after transforming it to first kind format.

\subsection{Performance with the $1^{\text {st }}$ order ODE examples from Kamke}

Although the main purpose of this paper is to present a computational scheme for finding solutions to Abel ODEs, it is interesting to see how odsolve - the ODE-solver of the ODEtools Maple package [12] - performs with the addition of these new routines. The performance with all of Kamke's 553 solvable examples 8 after incorporating the computational routines presented in this paper is: $97 \%$ are solved. This performance is summarized as follows

\begin{tabular}{|c|c|c|c|c|}
\hline \multirow{2}{*}{ Degree in $y^{\prime}$} & \multirow{2}{*}{ ODEs } & \multirow{2}{*}{ Solved } & \multicolumn{2}{|c|}{ Average time } \\
\cline { 4 - 5 } & 350 & 337 & 3.2 sec. & 12.9 sec. \\
\hline 1 & 145 & 140 & 8.8 sec. & 61.1 sec. \\
\hline 2 & 27 & 26 & 7.2 sec. & 17 sec. \\
\hline 3 & 31 & 30 & 13.4 sec. & 25.2 sec. \\
\hline higher & 553 & 533 & $\approx 6$ sec. & $\approx 20$ sec. \\
\hline \hline Total: & \multicolumn{2}{|c|}{ Table 8. Kamke's first order ODEs, solved by odsolve: $97 \%$}
\end{tabular}

The number and classification of Kamke's $1^{\text {st }}$ order ODEs still not solved by odsolve is now:

\begin{tabular}{|l|l|}
\hline Class & Kamke's numbering \\
\hline rational & 452 \\
\hline Riccati & 25 \\
\hline NONE & $80,81,83,87,121,128,340,367,395,460,506,510,543,572$ \\
\hline
\end{tabular}

Table 9. Kamke's $1^{\text {st }}$ order solvable ODEs for which odsolve fails: $3 \%$

where the Abel ODEs numbered in Kamke's book as 47, 48, 205, 206, 237 and 265 not presented in the tables above are known to be solvable only for specific values of their parameters - and for these values odsolve succeeds - and the ODEs 234 and 253 were not included since their solutions are not shown in the book or known to us.

\section{Conclusions}

In this paper, a first classification, according to invariant theory, of solvable non-constant invariant Abel ODEs found in the literature, was presented. Also, a set of new solvable classes, depending on one or no parameters, derived from the analysis of the works by Abel, Liouville and Appell [2, 23, 歫, was shown. Computer algebra routines were then developed, in the framework of the Maple ODEtools package, to solve - in principle - any member of these classes by solving its related equivalence problem. The result is a concrete new tool for solving Abel type ODEs fully integrated with Maple's ODE-solver dsolve.

The classification shown has had the intention of giving a first step towards organizing in a single reference the integrable cases widely scattered throughout the literature. The derivation of

\footnotetext{
${ }^{27}$ For Kamke's ODE 257, Macsyma (2.7) returns a wrong answer in terms of $y^{\prime}$.

${ }^{28}$ We classified as unsolvable in general Kamke's examples 50, 55, 56, 74, 79, 82, 202, 219, 250, 269, 331, 370, 461, 503 and 576.
} 
new solvable parameterized classes from the works by Abel and Appell in the $19^{\text {th }}$ century (Classes "C" and "D") also showed that valuable information can still be obtained from these old papers. In fact, from Tables 1, 2, 3 and 4 in sec. 14, the larger number of integrable cases found in textbooks are particular members of this Class "C" (Eq.(49) ) - an integrable class derived from a case somehow disregarded in Abel's Memoires [2].

As for the computer routines, the implementation presented here for solving the equivalence problem for parameterized classes proved to be a valuable tool in most of the Abel ODE examples we were able to collect. The Abel ODE routines here presented were recently integrated to the Maple computer algebra system and are already part of the upcoming Maple release 6.

On the other hand, we note the intrinsic limitation of this Abel ODE problem: most of the solutions can only be obtained in implicit form and in terms of quadratures; in turn, these integrals are usually elliptic integrals so that they cannot be expressed using elementary functions. Also the classification of integrable cases presented here is incomplete in that it is missing - at least - a more thorough analysis of the integrable cases presented in [11]. We are presently working on this topic and expect to succeed in obtaining reportable results in the near future.

\section{Acknowledgments}

This work was partially supported by the State University of Rio de Janeiro (UERJ), Brazil and by the Symbolic Computation Group, Faculty of Mathematics, University of Waterloo, Ontario, Canada. The authors would like to thank K. von Bülow for a careful reading of this paper, and T. Kolokolnikov and A. Wittkopf for fruitful discussions.

\section{References}

[1] E. Kamke, Differentialgleichungen: Lösungsmethoden und Lösungen. Chelsea Publishing Co, New York (1959).

[2] N.H. Abel, Oeuvres Complètes II, S.Lie and L.Sylow, Eds., Christiana, 1881.

[3] R. Liouville, "Sur une équation différentielle du premier ordre", Acta Mathematica 26, 55-78 (1902). See also R. Liouville, Comptes Rendus 103, 476-479 (1886) and R. Liouville, Comptes Rendus 460-463 (1887).

[4] P. Appell, "Sur les invariants de quelques équations différentielles", Journal de Mathématique 5, 361-423 (1889).

[5] G.M. Murphy, Ordinary Differential Equations and their solutions. Van Nostrand, Princeton, (1960).

[6] F. Schwarz, "Algorithmic Solution of Abel's Equation", Computing 61, 39-46 (1998).

[7] P. Olver, Equivalence, Invariants and Symmetry, Cambridge University Press (1995).

[8] K.O. Geddes, S.R. Czapor, G. Labahn, Algorithms for Computer Algebra, Kluwer Academic Publishers (1992).

[9] M. Chini, "Sull'integrazione di alcune equazioni differenziali del primo ordine", Rendiconti Instituto Lombardo (2) 57, 506-511 (1924).

[10] M. Halphen, "Sur la multiplication de fonctions elliptiques", Comptes Rendus T. LXXXVIII, $\mathrm{N}^{o} 9$ (1879). 
[11] A.D. Polyanin, V.F. Zaitsev, Handbook of Exact Solutions for Ordinary Differential Equations, CRC Press, Boca Raton (1995).

[12] E.S. Cheb-Terrab and A.D. Roche, "Integrating Factors for Second Order ODEs", Journal of Symbolic Computation V. 27, No. 5, p. 501-519 (1999); E.S. Cheb-Terrab, A.D. Roche "Symmetries and first order ODE patterns", Computer Physics Communications 113 (1998) 239; E.S. Cheb-Terrab, L.G.S. Duarte and L.A.C.P. da Mota, "Computer Algebra Solving of Second Order ODEs Using Symmetry Methods", Computer Physics Communications, 108 (1998); E.S. ChebTerrab, L.G.S. Duarte and L.A.C.P. da Mota, "Computer Algebra Solving of First Order ODEs Using Symmetry Methods", Computer Physics Communications 101 (1997).

[13] F. Boulier, D. Lazard, F. Ollivier and M. Petitot "Representation for the radical of a finitely generated differential ideal", Proc. ISSAC 1995, ACM Press 158-166 (1995).

[14] G.J. Reid, A.D. Wittkopf and A. Boulton, "Reduction of systems of nonlinear partial differential equations to simplified involutive forms", Eur. J. Appl. Math. 7 604-635 (1996). 


\section{Appendix A}

\begin{tabular}{|c|c|}
\hline Class & Representative equation and solution \\
\hline 1 & $y^{\prime}=\frac{3 y^{2}-3 y-x}{x(8 y-9)}, \quad C_{1}+\frac{x^{3}\left(4 x^{2}+\left(8 y^{2}-36 y+27\right) x+4 y^{4}-4 y^{3}\right)}{\left(x^{2}+2 x\left(y^{2}-3 y\right)+y^{4}\right)^{3}}=0$ \\
\hline 2 & $y^{\prime}=-2 y^{2} x+y^{3}, \quad C_{1}+\frac{x \operatorname{Ai}\left(x^{2}-\frac{1}{y}\right)+\operatorname{Ai}\left(1, x^{2}-\frac{1}{y}\right)}{x \operatorname{Bi}\left(x^{2}-\frac{1}{y}\right)+\operatorname{Bi}\left(1, x^{2}-\frac{1}{y}\right)}=0$ \\
\hline 3 & $y^{\prime}=\frac{y^{3}}{4 x^{2}}-y^{2}, \quad C_{1}+\frac{\left(x-\frac{1}{y}\right) \operatorname{Ai}\left(\left(x-\frac{1}{y}\right)^{2}-\frac{1}{2 x}\right)+\mathrm{Ai}\left(1,\left(x-\frac{1}{y}\right)^{2}-\frac{1}{2 x}\right)}{\left(x-\frac{1}{y}\right) \operatorname{Bi}\left(\left(x-\frac{1}{y}\right)^{2}-\frac{1}{2 x}\right)+\operatorname{Bi}\left(1,\left(x-\frac{1}{y}\right)^{2}-\frac{1}{2 x}\right)}=0$ \\
\hline 4 & $y^{\prime}=y^{3}-\frac{x+1}{x} y^{2}, \quad C_{1}+\frac{1}{x} e^{\frac{1}{y}-x}-\operatorname{Ei}\left(1, x-\frac{1}{y}\right)=0$ \\
\hline 5 & 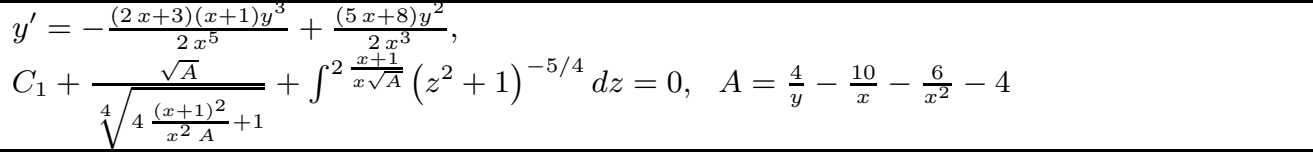 \\
\hline 6 & $y^{\prime}=-\frac{y^{3}}{x^{2}(x-1)^{2}}+\frac{\left(1-x-x^{2}\right) y^{2}}{x^{2}(x-1)^{2}}, \quad C_{1}-\operatorname{Ei}\left(1, \frac{y+x^{2}-x}{x y(x-1)}\right)+\frac{(x-1) y \mathrm{e}^{\frac{x-y-x^{2}}{x y(x-1)}}}{x-1+y}=0$ \\
\hline 7 & $\begin{array}{l}y^{\prime}=\frac{\left(4 x^{4}+5 x^{2}+1\right) y^{3}}{2 x^{3}}+y^{2}+\frac{\left(1-4 x^{2}\right) y}{2 x\left(x^{2}+1\right)} \\
C_{1}+2 \frac{x+A}{\sqrt[4]{A^{2}+1}(A x-1)}+\int^{A}\left(z^{2}+1\right)^{-5 / 4} d z=0 \quad A=\frac{x-2 y x^{4}-3 y x^{2}-y}{x\left(x+y x^{2}+y\right)}\end{array}$ \\
\hline A & $\begin{array}{l}y^{\prime}=\left(\alpha x+\frac{1}{x}+\frac{1}{x^{3}}\right) y^{3}+y^{2} \\
C_{1}+\frac{x^{3}}{y+x} \exp \left(\int^{\frac{-y x^{2}}{y+x}} \frac{2 d z}{z^{2}-z-\alpha z^{3}}\right)-\int^{\frac{-y x^{2}}{y+x}} \exp \left(\int \frac{2 d z}{z^{2}-z-\alpha z^{3}}\right) d z=0\end{array}$ \\
\hline B & $\begin{array}{l}y^{\prime}=2\left(x^{2}-\alpha^{2}\right) y^{3}+2(x+1) y^{2} \\
C_{1}+\frac{(\alpha+x) \mathrm{K}\left(\alpha,-\sqrt{x^{2}+\frac{1}{y}-\alpha^{2}}\right)+\sqrt{x^{2}+\frac{1}{y}-\alpha^{2}} \mathrm{~K}\left(1+\alpha,-\sqrt{x^{2}+\frac{1}{y}-\alpha^{2}}\right)}{(\alpha+x) \mathrm{I}\left(\alpha,-\sqrt{x^{2}+\frac{1}{y}-\alpha^{2}}\right)-\sqrt{x^{2}+\frac{1}{y}-\alpha^{2}} \mathrm{I}\left(1+\alpha,-\sqrt{x^{2}+\frac{1}{y}-\alpha^{2}}\right)}=0\end{array}$ \\
\hline $\mathrm{C}$ & $y^{\prime}=\frac{\alpha\left(1-x^{2}\right) y^{3}}{2 x}+(\alpha-1) y^{2}-\frac{\alpha y}{2 x}, \quad C_{1}+\frac{\alpha}{x}\left(1-\left(\frac{1}{y}-x\right)^{2}\right)^{1 / \alpha}-2 \int^{\frac{1-x y}{y}}\left(1-z^{2}\right)^{\frac{1-\alpha}{\alpha}} d z=0$ \\
\hline $\mathrm{D}$ & $\begin{array}{l}y^{\prime}=-\frac{y^{3}}{x}-\frac{\left(\alpha+x^{2}\right) y^{2}}{x^{2}} \\
C_{1}+\frac{(\alpha+1) \mathrm{M}\left(-\frac{\alpha}{2}-\frac{3}{4}, \frac{1}{4}, \frac{1}{2}\left(x-\frac{\alpha}{x}-\frac{1}{y}\right)^{2}\right)+\left(\frac{x}{y}-x^{2}\right) \mathrm{M}\left(-\frac{\alpha}{2}+\frac{1}{4}, \frac{1}{4}, \frac{1}{2}\left(x-\frac{\alpha}{x}-\frac{1}{y}\right)^{2}\right)}{\left(\alpha^{2}+\alpha\right) \mathrm{W}\left(-\frac{\alpha}{2}-\frac{3}{4}, \frac{1}{4}, \frac{1}{2}\left(x-\frac{\alpha}{x}-\frac{1}{y}\right)^{2}\right)+2\left(\frac{x}{y}-x^{2}\right) \mathrm{W}\left(-\frac{\alpha}{2}+\frac{1}{4}, \frac{1}{4}, \frac{1}{2}\left(x-\frac{\alpha}{x}-\frac{1}{y}\right)^{2}\right)}=0\end{array}$ \\
\hline
\end{tabular}

Representative ODEs and their solutions for the Abel ODE classes presented in this work.

\footnotetext{
${ }^{29}$ The solution shown for the representative of class D is not valid when $\alpha$ is an integer, or when $2 \alpha$ is a positive integer. In those cases, the solution of the associated Riccati equation Eq.(55) takes many different forms depending on the value of $\alpha$, which we found inconvenient to present here. $\operatorname{Ei}(n, x)=\int_{1}^{\infty} \frac{\mathrm{e}^{-x t}}{t^{n}} d t$ is the exponential integral, $\operatorname{Ai}(x)$ and $\operatorname{Bi}(x)$ are the Airy wave functions, $\mathrm{K}(x)$ and $\mathrm{I}(x)$ are the modified Bessel functions of the first and second kinds, respectively, and $\mathrm{M}(x)$ and $\mathrm{W}(x)$ are the Whittaker functions.
} 